Epidemiology and Psychiatric

Sciences

cambridge.org/eps

\section{Original Article}

Cite this article: Luo W, Zhong B-L, Chiu HF-K (2021). Prevalence of depressive symptoms among Chinese university students amid the COVID-19 pandemic: a systematic review and meta-analysis. Epidemiology and Psychiatric Sciences 30, e31, 1-21. https://doi.org/10.1017/ S2045796021000202

Received: 8 September 2020

Revised: 26 February 2021

Accepted: 8 March 2021

\section{Key words:}

COVID-19; depressive symptoms; metaanalysis; prevalence; systematic review; university students

Author for correspondence: Bao-Liang Zhong,

E-mail: haizhilan@gmail.com

\title{
Prevalence of depressive symptoms among Chinese university students amid the COVID-19 pandemic: a systematic review and meta-analysis
}

\section{Wei Luo ${ }^{1}$, Bao-Liang Zhong1,2 (D) and Helen Fung-Kum Chiu ${ }^{3}$}

${ }^{1}$ Research Center for Psychological and Health Sciences, China University of Geosciences, Wuhan, China;
${ }^{2}$ Affiliated Wuhan Mental Health Center, Tongji Medical College of Huazhong University of Science \& Technology,
Wuhan, China and ${ }^{3}$ Department of Psychiatry, The Chinese University of Hong Kong, Hong Kong SAR, China

\begin{abstract}
Aims. Chinese university students are at high risk for depressive symptoms and the ongoing coronavirus disease 2019 (COVID-19) pandemic may have exacerbated the mental health of university students. However, existing studies on depressive symptoms in Chinese university students during the COVID-19 pandemic reported a wide range of prevalence estimates, making mental health planning for this population difficult. The objective of this study was to conduct a systematic review and meta-analysis of surveys that assessed the prevalence of depressive symptoms in Chinese university students amid the COVID-19 pandemic.

Methods. Major Chinese (CNKI, Wanfang, VIP) and English (PubMed, Embase, PsycInfo) databases and preprint platforms were searched to identify cross-sectional studies containing data on the prevalence of depressive symptoms in Chinese university students during the pandemic. Two authors independently retrieved the literature, evaluated the eligibility of potential studies, assessed the risk of bias (RoB) of included studies, and extracted data. RoB was assessed with the Joanna Briggs Institute Critical Appraisal Checklist for Studies Reporting Prevalence Data. Results. In total, 1177 records were retrieved, and 84 studies involving 1292811 Chinese university students during the pandemic were included. None of the included studies were rated as completely low RoB. Statistically significant heterogeneity in the prevalence estimates of included studies was detected $\left(I^{2}=99.9 \%, p<0.001\right)$. The pooled prevalence of depressive symptoms was $26.0 \%$ (95\%CI: $23.3-28.9 \%)$, which was significantly higher in female than in male students $(30.8 \%$ v. $28.6 \%, p<0.001)$, in postgraduates than in undergraduates $(29.3 \%$ v. $22.9 \%, p<0.001)$, in students living inside than in those living outside the COVID-19 epicentre $(27.5 \% v .22 .3 \%, P<0.001)$, in students from universities at the epicentre than in those from universities outside the epicentre $(26.2 \% v .23 .1 \%, p<0.001)$, in students who had close contact with COVID-19 than in those who did not $(46.0 \% v .25 .0 \%, p<0.001)$, and in students who had acquaintances or relatives infected with COVID-19 (39.7\% v. 24.0\%, $p<0.001)$ than in those who did not. Five sources of heterogeneity were identified from the subgroup analysis: survey period, \% of males among the survey sample, scale of depressive symptoms, cutoff score of the scale and level of RoB.

Conclusions. Over one-fourth of Chinese university students experienced depressive symptoms during the COVID-19 pandemic. Mental health services for this population should include periodic evaluation of depressive symptoms, expanded social support and psychiatric assessment and treatment when necessary. It is also necessary to design depression prevention programmes that target higher-risk cohorts of university students.
\end{abstract}

\section{Introduction}

Studying in university is an important life stage during which a person moves from family dependence to independence and socialisation. The transition is challenging because of the high level of academic and employment stress and the prevalent interpersonal, romantic and emotional problems in this particular stage for university students (Zhao et al., 2015; Liu et al., 2017; Zhang et al., 2020a). However, due to China's strict examination-oriented education system, many university students have little training in interpersonal communication, problem solving and teamwork skills before entering university. Therefore, this population has difficulties in adapting to the university environment and is more likely to feel unconfident and confused about the future (Kirkpatrick and Zang, 2011; Hu, 2018). Moreover, university students in China have a high likelihood of experiencing parent-adolescent conflict owing to the popular authoritarian parenting style in the context of Chinese culture, which is characterised by high control and high warmth (Marmorstein and Iacono, 2004; Diao, 2007; Ren and Edwards, 2015). As a result, Chinese university students are at high risk for common 
mental health problems; for example, empirical evidence from a systematic review of 39 studies has shown that as high as $23.8 \%$ of Chinese university students suffer from depressive symptoms (Lei et al., 2016).

The ongoing coronavirus disease 2019 (COVID-19) pandemic has caused a global mental health crisis. Lessons learned from the 2003 severe acute respiratory syndrome (SARS) epidemic in China suggest that depressive symptoms are one of the most common mental health problems among university students; for example, during the SARS epidemic, $25.4-29.6 \%$ of the Chinese university students had depressive symptoms (Dang et al., 2004; Liu et al., 2004). In China, the pandemic has changed many aspects of university students' daily lives. Despite an increase in time spent with parents, home-isolated students have an increased chance of conflicting with parents (Luo, 2020). To prevent the spread of the epidemic, students are not allowed to return to campus to resume their studies, potentially delaying their graduation dates. Furthermore, because of social distancing and stay-at-home requirements, social and peer interactions are reduced, likely resulting in an increased level of social disconnectedness and a decreased level of peer support. Because parent-adolescent conflict, social disconnectedness and a lack of peer support have been associated with depressive symptoms in adolescents (Vaughan et al., 2010; Elmer and Stadtfeld, 2020; Rognli et al., 2020), the emotional health of Chinese university students may have been exacerbated by the COVID-19 pandemic.

Mental health services and crisis psychological intervention have been an essential part of the battle against the COVID-19 pandemic (Li et al., 2020a). To facilitate the development of population-specific intervention programmes, it is necessary to understand the epidemiology of depressive symptoms in university students in China amid the COVID-19 pandemic. However, available studies on depressive symptoms among Chinese university students have varied widely in terms of sampling methods, sample sizes and assessments of depressive symptoms, and most importantly, there have been considerable variations in the reported prevalence of depressive symptoms (1.8-79.3\%) (Liang et al., 2020a; Ren et al., 2020b), making mental health policymaking and planning difficult. To help clarify this issue, we performed a systematic review and meta-analysis on the prevalence of depressive symptoms among Chinese university students during the COVID-19 pandemic.

\section{Methods}

This systematic review and meta-analysis was reported in accordance with the Preferred Reporting Items for Systematic Reviews and Meta-Analyses (PRISMA) guidelines, and the protocol was registered in the International Prospective Register of Systematic Reviews (PROSPERO) with the registration number CRD 42020206666.

\section{Inclusion and exclusion criteria}

The inclusion criteria for eligible studies were (a) cross-sectional surveys or baseline surveys of cohort studies with meta-analysable data (i.e. reporting the prevalence of depressive symptoms); (b) study subjects were Chinese university students, including overseas students and postgraduates; (c) the presence of depressive symptoms was assessed with standardised instruments and (d) the study was conducted during the COVID-19 pandemic (since 1 January 2020). We excluded studies with mixed samples that did not present results separately for university students and studies that assessed depressive symptoms with unstandardised instruments (i.e. a simple self-designed question or a self-designed scale without convincing evidence of reliability and validity).

\section{Literature search}

We searched potential studies published between 1 January 2020 and 10 February 2021 in both Chinese and English bibliographic databases: China National Knowledge Infrastructure, Wanfang data, VIP Information, PubMed, Embase and PsycInfo. Key terms used were: (adolescen ${ }^{\star}$ OR teenager ${ }^{\star}$ OR youth ${ }^{\star}$ OR student ${ }^{\star}$ OR young adult ${ }^{\star}$ OR undergraduate ${ }^{\star}$ OR universit* OR college ${ }^{\star}$ ), (coronavirus disease 2019 or severe acute respiratory syndrome coronavirus 2 or COVID-19 or COVID) and (depress $\left.{ }^{\star}\right)$. To avoid missing relevant studies, reference lists of the retrieved reviews and included studies were also hand-searched. Preprint servers were also searched to retrieve grey literature: medRxiv, bioRxiv, PsyArXiv, ChinaXiv and Research Square. The literature search was ended on 12 February 2021. Detailed search strategies are provided in online Supplementary Table 1.

\section{Data extraction}

By using a predesigned electronic form, the following variables were extracted from included studies: first author, study site, study period, characteristics of the study sample, sampling method, sample size, survey method, assessment of depressive symptoms and rates of depressive symptoms. According to the State Council Information Office of the People's Republic of China (The State Council Information Office of the People's Republic of China, 2020), the study period in China was roughly classified as early stage of the COVID-19 outbreak (20 January-20 February 2020), late stage of the COVID-19 outbreak (21 February-28 April 2020) and post-COVID-19 outbreak (since 29 April 2020).

\section{RoB assessment of included studies}

We used the Joanna Briggs Institute (JBI) Critical Appraisal Checklist for Studies Reporting Prevalence Data (abbreviated as 'JBI checklist' hereafter) to assess the RoB of included studies (Munn et al., 2014). This checklist evaluates the RoB in terms of nine methodological domains: sample frame, sampling, sample size, description of subjects and setting, sample coverage of the data analysis, validity of the method for assessing the outcome, standardisation and reliability of the method for assessing outcome, statistical analysis and response rate. Two example items of the JBI checklist used in the current study were 'Was the sample size adequate?' and 'Were valid methods used for assessing depressive symptoms?'. Each item has four choices: yes, no, unclear or not applicable. One point is assigned to a 'yes' response, and the RoB score is the sum of the nine items, ranging from zero to nine, with a higher score indicating a lower RoB. In this study, the level of RoB of included studies was operationally categorised into low (RoB score of '7-9'), moderate (RoB score of '4-6') and high (RoB score of '0-3'). A RoB score of nine represents 'completely low RoB'.

Literature search, study inclusion, data extraction and RoB assessment were independently performed by the first and second authors of this study. They discussed their differences to arrive at a consensus when disagreement occurred in an assessment. 


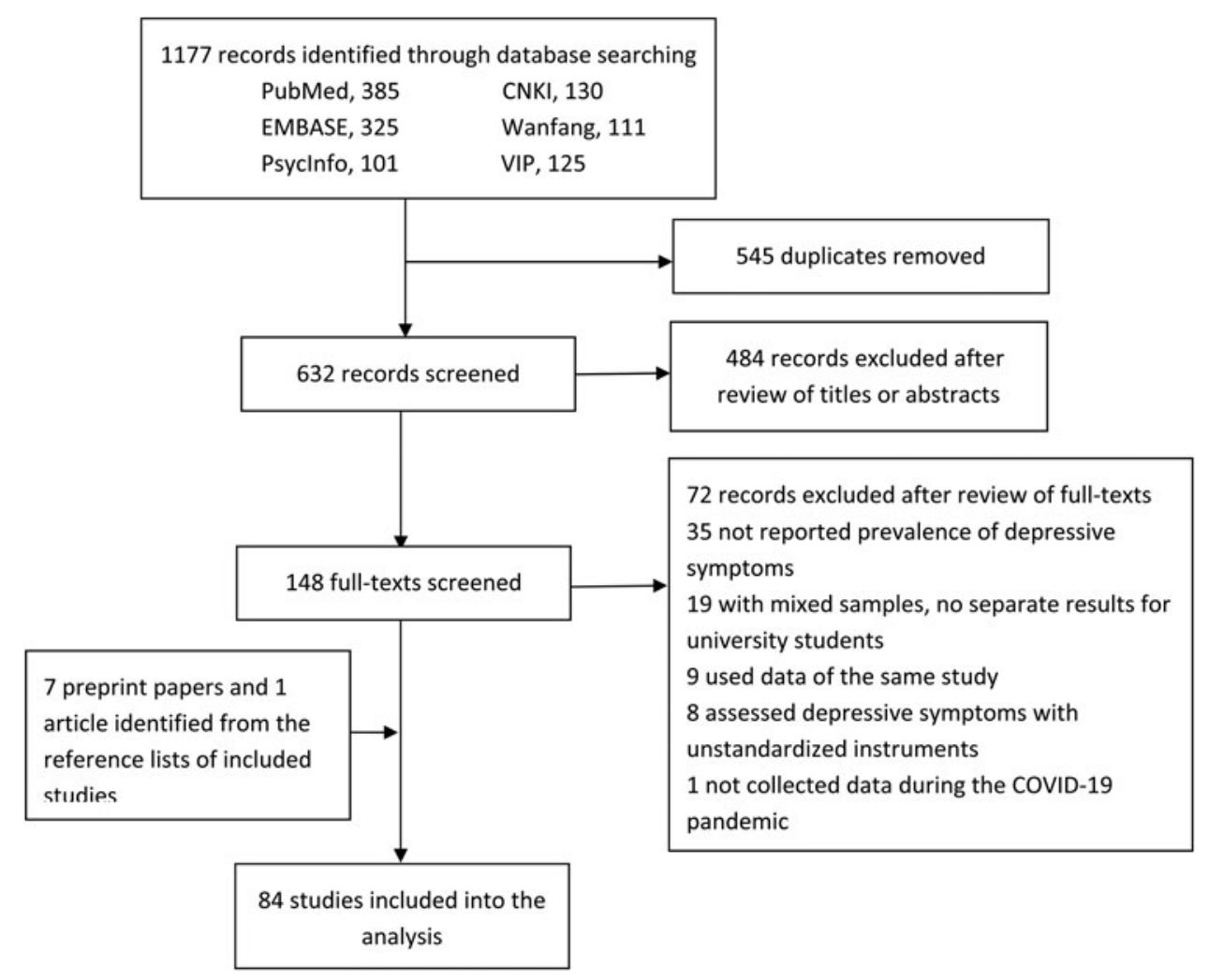

Fig. 1. Flowchart of study inclusion.

\section{Statistical analysis}

We used meta-analysis to generate pooled estimates and their $95 \%$ confidence intervals $(95 \% \mathrm{CIs})$ for the prevalence of depressive symptoms in the whole sample and in various cohorts of the sample. Forest plots were adopted to display the prevalence rates and pooled estimates. We used the $I^{2}$ test to evaluate heterogeneity between studies. When there was little evidence of heterogeneity (i.e. $I^{2} \leqslant 50 \%$, heterogeneity $P \geqslant 0.10$ ), a fixed-effect model was used to generate the pooled estimates; otherwise, the random-effect model was used. The pooled rates of various cohorts were compared by using the $Z$ test. We used subgroup analysis to explore the source of heterogeneity in the prevalence estimate of depressive symptoms. The $Q$-value test was used to test the significance of differences in prevalence rates between subgroups. Publication bias was assessed with funnel plots and Begg's test, since Begg's test is fairly powerful for large meta-analyses that include 75 or more original studies (Begg and Mazumdar, 1994). Before pooled analysis, prevalence proportions were transformed by using the Freeman-Tukey variant of the arcsine square root, Arcsine, untransformed, Log or Logit, as appropriate (Barendregt et al., 2013). All analyses were conducted using $\mathrm{R}$ (version 4.0.2). A two-sided $P<0.05$ was considered statistically significant.

\section{Results}

\section{Characteristics of included studies}

The process of study inclusion is shown in Fig. 1. Finally, this meta-analysis included 84 studies with a total of 1292811 Chinese university students (Cao, 2020; Chang et al., 2020;
Chen et al., 2020a, 2020b, 2020c, 2020d; Chi et al., 2020; Cong et al., 2020; Deng et al., 2020; Dong, 2020; Dong et al., 2020; Feng, 2020; Feng et al., 2020; Han et al., 2020; Ji et al., 2020; Jiang et al., 2020; Lei et al., 2020; Li and He, 2020; Li et al., 2020b; Lian et al., 2020; Liang et al., 2020a, 2020b; Lin and Xu, 2020; Lin et al., 2020a, 2020b; Liu, 2020a, 2020b; Liu et al., 2020a, 2020b, 2020c; Ma et al., 2020a, 2020b; Mao et al., 2020; Qian, 2020; Ren et al., 2020a, 2020b; 2020c; Si et al., 2020; Sun et al., 2020, 2021; Tang et al., 2020; Wan and Shao, 2020; Wang and $\mathrm{He}, 2020$; Wang and $\mathrm{Li}, 2020$; Wang et al., 2020b; 2020c; 2020d; 2020e; 2020f; 2021; Wei, 2020; Wu et al., 2020, 2021; Xiang et al., 2020; Xiao et al., 2020a, 2020b; Xie et al., 2020; Xin et al., 2020; Xing et al., 2020; Xiong et al., 2020; Xu and Li, 2020; Yan et al., 2020; Yang et al., 2020b; Yao et al., 2020; Yi et al., 2020a, 2020b; Yu et al., 2020, 2021; Zhan et al., 2020; Zhang et al., 2020b, 2020c, 2020d, 2020e, 2020f, 2020g; 2020h; Zhao and Hu, 2020; Zhao et al., 2020a, 2020b, 2020c; Zhou et al., 2020; Chen and Zhu, 2021; Ni et al., 2021; Pan et al., 2021). Among the 84 studies, seven were preprint articles (Cong et al., 2020; Liu et al., 2020c; Si et al., 2020; Xiong et al., 2020; Zhang et al., 2020h; Zhao et al., 2020b; Zhou et al., 2020), eight had samples recruited from universities at China's COVID-19 epicentre (Hubei or Wuhan) (Deng et al., 2020; Liu et al., 2020a; Wang et al., 2020d, 2020e; Xiao et al., 2020b, 2020a; Xu and Li, 2020; Wu et al., 2021) and two recruited samples of overseas Chinese students (Cong et al., 2020; Zhao et al., $2020 b$ ). A total of 23 studies adopted probability sampling to recruit subjects, while the remaining studies adopted convenience sampling. The sample sizes of included studies ranged between 84 and 746217 , with a median of 973 . A vast majority of the studies collected data via online self-administered questionnaires, while 
seven collected data via paper-pencil self-administered questionnaires (Chen et al., 2020a, 2020c, 2020d; Dong et al., 2020; Liu, 2020b; Liu et al., 2020b; Wu et al., 2020). Among the included studies, the Nine-item Patient Health Questionnaire (PHQ-9) was the most common instrument to assess the presence of depressive symptoms $(n=37)$, followed by Zung's Self-rating Depression Scale (SDS) $(n=22)$, the depression subscale of the Symptom Checklist-90-Revised (SCL-90-R) $(n=8)$, the depression subscale of the Depression, Anxiety and Stress Scale - 21 Items (DASS-21) $(n=7)$ and the Center for Epidemiologic Studies - Depression Scale $($ CES-D) $(n=7)$. The average and median reported prevalence rates of depressive symptoms were $27.3 \%$ and $25.8 \%$, respectively. Other detailed characteristics of the included studies are shown in Table 1.

\section{RoB of included studies}

In total, 31 studies had a RoB score of ' $0-3$ ', 42 had a RoB score of ' $4-6$ ' and 11 had a RoB score of ' $7-8$ '. No study was scored nine. The two most common methodological issues were inappropriate sample frame $(n=62)$ and problematic sampling method $(n=58)$ (online Supplementary Table 2).

\section{Meta-analysis of prevalence of depressive symptoms}

The pooled prevalence of depressive symptoms among Chinese university students was $26.0 \%$ (\%CI: 23.3-28.9\%) (Fig. 2). Pooled prevalence rate of severe depressive symptoms was 1.69\% (95\%CI: 0.87-2.77\%) (Fig. 3).

The combined prevalence rates of depressive symptoms were significantly higher in female than in male students $(30.8 \% v$. $28.6 \%, p<0.001)$, in students with siblings than in only child students $(24.2 \%$ v. $20.7 \%, p<0.001)$, in overseas than in domestic students $(44.5 \%$ v. $25.6 \%, p<0.001)$, in postgraduates than in undergraduates $(29.3 \%$ v. $22.9 \%, p<0.001)$, in students living in Hubei than in those living in provinces other than Hubei $(27.5 \%$ v. $22.3 \%, p<0.001)$, in students from universities of Hubei than in those from universities of other provinces $(26.2 \%$ v. $23.1 \%, p<0.001)$, in students who were in close contact with COVID-19 than in those who had no history of COVID-19 contact $(46.0 \%$ v. $25.0 \%, p<0.001)$, and in students who had friends, classmates or relatives infected with COVID-19 than in those who did not $(39.7 \%$ v. $24.0 \%, p<$ 0.001) (Table 2).

\section{Publication bias among included studies}

As shown in Fig. 4, the funnel plot was generally symmetric. The $p$ value of the Begg's test was 0.169 . No statistically significant publication bias was detected across the 84 included studies.

\section{Source of heterogeneity}

Five factors were identified as sources of heterogeneity across included studies (Table 3): survey period, \% of male students among the total sample, scale of depressive symptoms, cutoff score of the scale of depressive symptoms and level of RoB. Specifically, significantly higher pooled prevalence rates of depressive symptoms were observed in studies conducted during the late stage of the COVID-19 outbreak than in those conducted during the early stage $(31.0 \%$ v. $21.8 \%, p=0.015)$, in studies with a percentage of males $<50 \%$ than in those with a percentage of males $\geqslant 50 \%(27.3 \% v .20 .6 \%, p=0.033)$, in studies assessing depressive symptoms with CES-D than in those using SCL-90-R (40.0\% v. $11.5 \%, p=0.002)$, in studies defining the presence of depressive symptoms as 'PHQ- $9 \geqslant 5$ ' than in those defining it as 'PHQ-9 $\geqslant 10^{\prime}(29.2 \%$ v. $15.5 \%, p<0.001)$, and in studies with a high RoB than in those with a low RoB $(28.4 \%$ v. $20.6 \%, p=0.011$ ).

\section{Discussion}

\section{Main findings}

This systematic review and meta-analysis summarised studies estimating the prevalence of depressive symptoms among Chinese university students amid the COVID-19 pandemic. We found an overall prevalence rate of $26.0 \%$ of depressive symptoms in Chinese university students and significantly higher rates in female students ( $v$. males), in students with siblings ( $v$. only children), in overseas students ( $v$. domestic), in postgraduates ( $v$. undergraduates), in students living within the COVID-19 epicentre ( $v$. those living outside), in students from universities at the epicentre ( $v$. those from universities of provinces other than Hubei), in close contacts of COVID-19-infected persons ( $v$. those without a history of COVID-19 contact) and in students who had COVID-19-infected friends, classmates or relatives ( $v$. those who did not). In addition, $1.69 \%$ of Chinese university students had severe depressive symptoms.

Compared to the $23.8 \%$ prevalence of depressive symptoms among Chinese university students during the non-COVID-19 era (Lei et al., 2016), a higher prevalence of depressive symptoms (26.0\%) was found in Chinese university students amid the COVID-19 pandemic. Nevertheless, the absolute difference between the two rates $(2.2 \%)$ is not very large in magnitude. We argue that the result from this direct comparison should be considered with caution because of the significant heterogeneity in the methodologies of included studies. As shown in Table 3, the pooled prevalence of depressive symptoms rose to $29.2 \%$ when included studies were restricted to those defining the presence of depressive symptoms as 'PHQ-9 $\geqslant 5$ '. Previously, empirical studies have reported that the prevalence rates of depressive symptoms in Chinese university students were 19.2\% (PHQ-9 $\geqslant$ 5), $7.8-12.6 \%$ (PHQ-9 $\geqslant 10$ ) and 26.9\% (CES-D $\geqslant 16$ ) (He et al., 2014; Wu, 2019; Zhao et al., 2019; Gao et al., 2020b; Leung et al., 2020; Li et al., 2021), which are all lower than the corresponding figures in our study $(29.2 \%, 15.5 \%$ and $40.0 \%$, Table 3). Moreover, the $1.69 \%$ prevalence of severe depressive symptoms in our study was higher than that reported in two previous studies with samples of Chinese university students (0.50.9\%) (Ma et al., 2019; Zhao et al., 2019). These data suggest an elevated risk of depressive symptoms in Chinese university students during the COVID-19 pandemic.

In addition to the abovementioned postponement of graduation, home quarantine and social disconnectedness due to the COVID-19 pandemic, the cooccurring 'infodemic' may also explain the elevated risk of depressive symptoms in university students. This is because smartphone and social media use are very popular among Chinese university students, and students are more likely to be exposed to negative information or even rumours from social media platforms such as short videos of overcrowded hospitals, physically and emotionally exhausted physicians and helpless infected patients. As a supporting case, in this pandemic, Chinese researchers have found the significant 


\begin{tabular}{|c|c|c|c|c|c|c|c|c|c|}
\hline Study & Subjects and setting & $\begin{array}{l}\text { Dates of the } \\
\text { survey }\end{array}$ & $\begin{array}{l}\text { Sampling } \\
\text { method }\end{array}$ & Sample size & $\begin{array}{c}\text { Male students, } \\
n(\%)\end{array}$ & Age (years) & Survey method & $\begin{array}{l}\text { Assessment of } \\
\text { depressive } \\
\text { symptoms }\end{array}$ & $\begin{array}{l}\text { Depressed } \\
\text { students, } n \\
\quad(\%)\end{array}$ \\
\hline Cao (2020) & $\begin{array}{l}\text { Undergraduates of a } \\
\text { junior college in Xi'an, } \\
\text { China }\end{array}$ & NR & $\begin{array}{l}\text { Convenience } \\
\text { sampling }\end{array}$ & 2733 & $1684(61.6)$ & Range: $16-24$ & $\begin{array}{l}\text { Online } \\
\text { self-administered } \\
\text { questionnaire }\end{array}$ & PHQ-9 $\geqslant 5$ & $575(21.0)$ \\
\hline $\begin{array}{l}\text { Chang } \\
\text { et al. } \\
(2020)\end{array}$ & $\begin{array}{l}\text { University students in } \\
\text { Guangdong, China }\end{array}$ & $\begin{array}{l}31 \text { January-3 } \\
\text { February } 2020\end{array}$ & $\begin{array}{l}\text { Convenience } \\
\text { sampling }\end{array}$ & 3881 & $1434(36.9)$ & Mean: 20.0 & $\begin{array}{l}\text { Online } \\
\text { self-administered } \\
\text { questionnaire }\end{array}$ & PHQ-9 $\geqslant 5$ & $821(21.2)$ \\
\hline $\begin{array}{l}\text { Chen et al. } \\
(2020 a)\end{array}$ & $\begin{array}{l}\text { Medical postgraduates } \\
\text { of a general hospital in } \\
\text { Hangzhou, China }\end{array}$ & February 2020 & $\begin{array}{l}\text { Convenience } \\
\text { sampling }\end{array}$ & 795 & $343(43.1)$ & Mean: 26.6 & $\begin{array}{l}\text { Paper-pencil } \\
\text { self-administered } \\
\text { questionnaire }\end{array}$ & PHQ-9 $\geqslant 5$ & $172(21.6)$ \\
\hline $\begin{array}{l}\text { Chen et al. } \\
(2020 b)\end{array}$ & $\begin{array}{l}\text { Undergraduates of } 85 \\
\text { universities in } \\
\text { Guangdong, China }\end{array}$ & $\begin{array}{l}13-22 \text { February } \\
2020\end{array}$ & $\begin{array}{l}\text { Convenience } \\
\text { sampling }\end{array}$ & 323489 & $130516(40.3)$ & 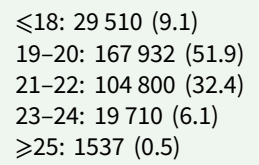 & $\begin{array}{l}\text { Online } \\
\text { self-administered } \\
\text { questionnaire }\end{array}$ & PHQ-9 $\geqslant 10$ & $24909(7.7)$ \\
\hline $\begin{array}{l}\text { Chen et al. } \\
(2020 c)\end{array}$ & $\begin{array}{l}\text { Medical postgraduates } \\
\text { of a general hospital in } \\
\text { Hangzhou, China }\end{array}$ & $\begin{array}{l}3-16 \text { February } \\
2020\end{array}$ & $\begin{array}{l}\text { Cluster } \\
\text { sampling }\end{array}$ & 286 & NR & NR & $\begin{array}{l}\text { Paper-pencil } \\
\text { self-administered } \\
\text { questionnaire }\end{array}$ & PHQ-9 $\geqslant 5$ & $54(18.9)$ \\
\hline $\begin{array}{l}\text { Chen et al. } \\
(2020 d)\end{array}$ & $\begin{array}{l}\text { Undergraduates and } \\
\text { postgraduates in } \\
\text { Beijing, China }\end{array}$ & $\begin{array}{l}\text { 'post-epidemic } \\
\text { of CPVID-19' }\end{array}$ & $\begin{array}{l}\text { Stratified } \\
\text { random } \\
\text { sampling }\end{array}$ & 697 & $183(26.3)$ & Mean: 24.3 & $\begin{array}{l}\text { Paper-pencil } \\
\text { self-administered } \\
\text { questionnaire }\end{array}$ & $\begin{array}{l}\text { Depression } \\
\text { subscale of } \\
\text { SCL-90-R }>2\end{array}$ & $61(8.8)$ \\
\hline $\begin{array}{l}\text { Chi et al. } \\
(2020)\end{array}$ & $\begin{array}{l}\text { University students in } \\
\text { China }\end{array}$ & $\begin{array}{l}12-17 \text { February } \\
2020\end{array}$ & $\begin{array}{l}\text { Convenience } \\
\text { sampling }\end{array}$ & 2038 & $755(37.0)$ & Mean: 20.6 & $\begin{array}{l}\text { Online } \\
\text { self-administered } \\
\text { questionnaire }\end{array}$ & PHQ-9 $\geqslant 10$ & $475(23.3)$ \\
\hline $\begin{array}{l}\text { Cong et al. } \\
(2020)\end{array}$ & $\begin{array}{l}\text { Oversea Chinese } \\
\text { undergraduates and } \\
\text { postgraduates }\end{array}$ & May $18-21,2020$ & $\begin{array}{l}\text { Convenience } \\
\text { sampling }\end{array}$ & 252 & $102(40.5)$ & $\begin{array}{l}<18: 2(0.8) \\
\text { 18-25: } 160(63.5) \\
\text { 26-30: } 70(27.8) \\
>30: 20(7.9)\end{array}$ & $\begin{array}{l}\text { Online } \\
\text { self-administered } \\
\text { questionnaire }\end{array}$ & PHQ-9 $\geqslant 5$ & $152(60.3)$ \\
\hline $\begin{array}{l}\text { Deng et al. } \\
(2020)\end{array}$ & $\begin{array}{l}\text { Undergraduates in } \\
\text { China }\end{array}$ & 8-11 May 2020 & $\begin{array}{l}\text { Convenience } \\
\text { sampling }\end{array}$ & 1607 & $1041(64.8)$ & $\begin{array}{l}<18: 20(1.2) \\
\text { 18-22: } 1573(97.9) \\
>22: 14(0.9)\end{array}$ & $\begin{array}{l}\text { Online } \\
\text { self-administered } \\
\text { questionnaire }\end{array}$ & $\begin{array}{l}\text { Depression } \\
\text { subscale of } \\
\text { DASS21 } \geqslant 10\end{array}$ & $56(3.5)$ \\
\hline $\begin{array}{l}\text { Dong et al. } \\
\text { (2020) }\end{array}$ & $\begin{array}{l}\text { Medical postgraduates } \\
\text { of a general hospital in } \\
\text { China }\end{array}$ & $\begin{array}{l}20 \text { January-20 } \\
\text { February } 2020\end{array}$ & $\begin{array}{l}\text { Convenience } \\
\text { sampling }\end{array}$ & 162 & $52(32.1)$ & Mean: 26.4 & $\begin{array}{l}\text { Paper-pencil } \\
\text { self-administered } \\
\text { questionnaire }\end{array}$ & $S D S \geqslant 53$ & $63(38.9)$ \\
\hline $\begin{array}{l}\text { Dong } \\
(2020)\end{array}$ & $\begin{array}{l}\text { Undergraduates of a } \\
\text { university in Linfen, } \\
\text { China }\end{array}$ & NR & $\begin{array}{l}\text { Cluster } \\
\text { sampling }\end{array}$ & 4085 & $923(22.6)$ & Mean: 18.9 & $\begin{array}{l}\text { Online } \\
\text { self-administered } \\
\text { questionnaire }\end{array}$ & $\begin{array}{l}\text { Depression } \\
\text { subscale of } \\
\mathrm{SCL}-90-\mathrm{R}>2\end{array}$ & $554(13.6)$ \\
\hline $\begin{array}{l}\text { Feng et al. } \\
(2020)\end{array}$ & $\begin{array}{l}\text { Students of a university } \\
\text { in Beijing, China }\end{array}$ & $\begin{array}{l}8-28 \text { February } \\
2020\end{array}$ & $\begin{array}{l}\text { Simple cluster } \\
\text { sampling }\end{array}$ & 1346 & $364(27.0)$ & Mean: 19.8 & $\begin{array}{l}\text { Online } \\
\text { self-administered } \\
\text { questionnaire }\end{array}$ & PHQ-9 $\geqslant 5$ & 429 (31.9) \\
\hline $\begin{array}{l}\text { Feng } \\
(2020)\end{array}$ & $\begin{array}{l}\text { Undergraduates of a } \\
\text { junior college in } \\
\text { Qingyuan, China }\end{array}$ & $\begin{array}{l}18-22 \text { February } \\
2020\end{array}$ & $\begin{array}{l}\text { Random } \\
\text { sampling }\end{array}$ & 7157 & $2158(30.2)$ & Median: 20.1 & $\begin{array}{l}\text { Online } \\
\text { self-administered } \\
\text { questionnaire }\end{array}$ & PHQ-9 $\geqslant 5$ & $1956(27.3)$ \\
\hline
\end{tabular}


Table 1. (Continued.)

\begin{tabular}{|c|c|c|c|c|c|c|c|c|c|}
\hline Study & Subjects and setting & $\begin{array}{l}\text { Dates of the } \\
\text { survey }\end{array}$ & $\begin{array}{l}\text { Sampling } \\
\text { method }\end{array}$ & Sample size & $\begin{array}{c}\text { Male students, } \\
n(\%)\end{array}$ & Age (years) & Survey method & $\begin{array}{l}\text { Assessment of } \\
\text { depressive } \\
\text { symptoms }\end{array}$ & $\begin{array}{c}\text { Depressed } \\
\text { students, } n \\
(\%)\end{array}$ \\
\hline $\begin{array}{l}\text { Han et al. } \\
(2020)\end{array}$ & $\begin{array}{l}\text { Undergraduates and } \\
\text { postgraduates in China }\end{array}$ & $\begin{array}{l}22-24 \text { February } \\
2020\end{array}$ & $\begin{array}{l}\text { Convenience } \\
\text { sampling }\end{array}$ & 405 & $134(33.1)$ & NR & $\begin{array}{l}\text { Online } \\
\text { self-administered } \\
\text { questionnaire }\end{array}$ & $\begin{array}{l}\text { Depression } \\
\text { subscale of } \\
\text { DASS21 } \geqslant 10\end{array}$ & $178(44.0)$ \\
\hline $\begin{array}{l}\text { Ji et al. } \\
\text { (2020) }\end{array}$ & $\begin{array}{l}\text { Nursing } \\
\text { undergraduates of } \\
\text { seven universities in } \\
\text { Sichuan, China }\end{array}$ & $\begin{array}{l}14-19 \text { February } \\
2020\end{array}$ & $\begin{array}{l}\text { Cluster } \\
\text { sampling }\end{array}$ & 1013 & $139(13.7)$ & Mean: 20.0 & $\begin{array}{l}\text { Online } \\
\text { self-administered } \\
\text { questionnaire }\end{array}$ & SDS $\geqslant 50$ & $247(24.4)$ \\
\hline $\begin{array}{l}\text { Jiang et al. } \\
\text { (2020) }\end{array}$ & $\begin{array}{l}\text { Medical } \\
\text { undergraduates of a } \\
\text { university in China }\end{array}$ & $\begin{array}{l}27-29 \text { February } \\
2020\end{array}$ & $\begin{array}{l}\text { Cluster } \\
\text { sampling }\end{array}$ & 399 & $162(40.6)$ & NR & $\begin{array}{l}\text { Online } \\
\text { self-administered } \\
\text { questionnaire }\end{array}$ & PHQ-9 $\geqslant 5$ & $104(26.1)$ \\
\hline $\begin{array}{l}\text { Lei et al. } \\
(2020)\end{array}$ & $\begin{array}{l}\text { Medical } \\
\text { undergraduates and } \\
\text { postgraduates of a } \\
\text { university in Tangshan, } \\
\text { China }\end{array}$ & NR & $\begin{array}{l}\text { Convenience } \\
\text { sampling }\end{array}$ & 231 & $109(47.2)$ & NR & $\begin{array}{l}\text { Online } \\
\text { self-administered } \\
\text { questionnaire }\end{array}$ & $S D S \geqslant 53$ & $143(61.9)$ \\
\hline $\begin{array}{l}\text { Li and He } \\
(2020)\end{array}$ & $\begin{array}{l}\text { Students of a junior } \\
\text { college in Jinhua, China }\end{array}$ & $\begin{array}{l}30 \text { January-15 } \\
\text { February } 2020\end{array}$ & $\begin{array}{l}\text { Convenience } \\
\text { sampling }\end{array}$ & 1144 & $597(52.2)$ & NR & $\begin{array}{l}\text { Online } \\
\text { self-administered } \\
\text { questionnaire }\end{array}$ & $\mathrm{PHQ}-9 \geqslant 5$ & $240(21.0)$ \\
\hline $\begin{array}{l}\text { Li et al. } \\
(2020 b)\end{array}$ & $\begin{array}{l}\text { Undergraduates of a } \\
\text { university in Chengdu, } \\
\text { China }\end{array}$ & $\begin{array}{l}\text { February-March } \\
2020\end{array}$ & $\begin{array}{l}\text { Cluster } \\
\text { sampling }\end{array}$ & 7747 & 3947 (50.9) & Mean: 20.7 & $\begin{array}{l}\text { Online } \\
\text { self-administered } \\
\text { questionnaire }\end{array}$ & $\mathrm{PHQ}-9 \geqslant 5$ & $1278(16.5)$ \\
\hline $\begin{array}{l}\text { Lian et al. } \\
(2020)\end{array}$ & $\begin{array}{l}\text { Undergraduates and } \\
\text { postgraduates of a } \\
\text { university in Changsha, } \\
\text { China }\end{array}$ & NR & $\begin{array}{l}\text { Random } \\
\text { sampling }\end{array}$ & 1437 & 789 (54.9) & NR & $\begin{array}{l}\text { Online } \\
\text { self-administered } \\
\text { questionnaire }\end{array}$ & $\begin{array}{l}\text { Depression } \\
\text { subscale of } \\
\text { SCL-90-R } \geqslant 2\end{array}$ & $177(12.3)$ \\
\hline $\begin{array}{l}\text { Liang et al. } \\
(2020 a)\end{array}$ & $\begin{array}{l}\text { Nursing junior college } \\
\text { students and nursing } \\
\text { undergraduates of } \\
\text { three universities in } \\
\text { Hebei, China }\end{array}$ & February 2020 & $\begin{array}{l}\text { Convenience } \\
\text { sampling }\end{array}$ & 852 & $80(9.4)$ & NR & $\begin{array}{l}\text { Online } \\
\text { self-administered } \\
\text { questionnaire }\end{array}$ & $\begin{array}{l}\text { Depression } \\
\text { subscale of } \\
S C L-90-R \geqslant 2\end{array}$ & $15(1.8)$ \\
\hline $\begin{array}{l}\text { Liang et al. } \\
(2020 b)\end{array}$ & $\begin{array}{l}\text { Medical postgraduates } \\
\text { of a general hospital in } \\
\text { Hangzhou, China }\end{array}$ & NR & $\begin{array}{l}\text { Convenience } \\
\text { sampling }\end{array}$ & 793 & $373(47.0)$ & NR & $\begin{array}{l}\text { Online } \\
\text { self-administered } \\
\text { questionnaire }\end{array}$ & $\begin{array}{l}\text { Depression } \\
\text { subscale of } \\
S C L-90-R \geqslant 2\end{array}$ & $40(5.0)$ \\
\hline $\begin{array}{l}\text { Lin et al. } \\
(2020 a)\end{array}$ & $\begin{array}{l}\text { Undergraduates and } \\
\text { postgraduates in China }\end{array}$ & $\begin{array}{l}10-16 \text { March } \\
2020\end{array}$ & $\begin{array}{l}\text { Convenience } \\
\text { sampling }\end{array}$ & 625 & $220(35.2)$ & Mean: 20.2 & $\begin{array}{l}\text { Online } \\
\text { self-administered } \\
\text { questionnaire }\end{array}$ & $C E S-D \geqslant 16$ & $217(34.7)$ \\
\hline $\begin{array}{l}\text { Lin et al. } \\
(2020 b)\end{array}$ & $\begin{array}{l}\text { Students of a medical } \\
\text { university in Fuzhou, } \\
\text { China }\end{array}$ & $15-20$ April 2020 & $\begin{array}{l}\text { Random } \\
\text { sampling }\end{array}$ & 320 & $149(46.6)$ & NR & $\begin{array}{l}\text { Online } \\
\text { self-administered } \\
\text { questionnaire }\end{array}$ & PHQ-9 > 5 & $183(57.2)$ \\
\hline $\begin{array}{l}\text { Lin and } X u \\
(2020)\end{array}$ & $\begin{array}{l}\text { Undergraduates of } \\
\text { universities in Fuzhou, } \\
\text { China }\end{array}$ & $\begin{array}{l}26-30 \text { March } \\
2020\end{array}$ & $\begin{array}{l}\text { Convenience } \\
\text { sampling }\end{array}$ & 1297 & $565(43.6)$ & NR & $\begin{array}{l}\text { Online } \\
\text { self-administered } \\
\text { questionnaire }\end{array}$ & PHQ-9 $\geqslant 5$ & $320(24.7)$ \\
\hline
\end{tabular}




\begin{tabular}{|c|c|c|c|c|c|c|c|c|c|}
\hline $\begin{array}{l}\text { Liu et al. } \\
(2020 a)\end{array}$ & $\begin{array}{l}\text { Undergraduates and } \\
\text { postgraduates of a } \\
\text { medical university in } \\
\text { Wuhan, China }\end{array}$ & $\begin{array}{l}23 \text { February-2 } \\
\text { April } 2020\end{array}$ & $\begin{array}{l}\text { Convenience } \\
\text { sampling }\end{array}$ & 217 & $90(41.5)$ & Mean: 21.7 & $\begin{array}{l}\text { Online } \\
\text { self-administered } \\
\text { questionnaire }\end{array}$ & $P H Q-9 \geqslant 5$ & $77(35.5)$ \\
\hline $\begin{array}{l}\text { Liu et al. } \\
(2020 b)\end{array}$ & $\begin{array}{l}\text { Undergraduates of a } \\
\text { medical university in } \\
\text { Beijing, China }\end{array}$ & NR & $\begin{array}{l}\text { Convenience } \\
\text { sampling }\end{array}$ & 611 & $198(32.4)$ & Range: $17-23$ & $\begin{array}{l}\text { Paper-pencil } \\
\text { self-administered } \\
\text { questionnaire }\end{array}$ & $S D S \geqslant 53$ & $101(16.5)$ \\
\hline $\begin{array}{l}\text { Liu et al. } \\
\text { (2020c) }\end{array}$ & $\begin{array}{l}\text { Junior college students, } \\
\text { undergraduates and } \\
\text { postgraduates in China }\end{array}$ & $\begin{array}{l}1-5 \text { February } \\
2020\end{array}$ & $\begin{array}{l}\text { Convenience } \\
\text { sampling }\end{array}$ & 509 & $176(34.6)$ & Mean: 21.3 & $\begin{array}{l}\text { Online } \\
\text { self-administered } \\
\text { questionnaire }\end{array}$ & $S D S \geqslant 50$ & $70(13.8)$ \\
\hline Liu (2020a) & $\begin{array}{l}\text { Undergraduates of a } \\
\text { university in Taiyuan, } \\
\text { China }\end{array}$ & $\begin{array}{l}20-22 \text { February } \\
2020\end{array}$ & $\begin{array}{l}\text { Convenience } \\
\text { sampling }\end{array}$ & 191 & NR & NR & $\begin{array}{l}\text { Online } \\
\text { self-administered } \\
\text { questionnaire }\end{array}$ & $\begin{array}{l}\text { Depression } \\
\text { subscale of } \\
\mathrm{SCL}-90-\mathrm{R}>2\end{array}$ & $14(7.3)$ \\
\hline Liu (2020b) & $\begin{array}{l}\text { Junior college students } \\
\text { and undergraduates in } \\
\text { Hangzhou, China }\end{array}$ & NR & $\begin{array}{l}\text { Convenience } \\
\text { sampling }\end{array}$ & 90 & $1(1.1)$ & Range: $20-23$ & $\begin{array}{l}\text { Paper-pencil } \\
\text { self-administered } \\
\text { questionnaire }\end{array}$ & $S D S \geqslant 53$ & $29(32.2)$ \\
\hline $\begin{array}{l}\text { Ma et al. } \\
(2020 a)\end{array}$ & $\begin{array}{l}\text { Undergraduates of a } \\
\text { university in Taiyuan, } \\
\text { China }\end{array}$ & $\begin{array}{l}10-15 \text { February } \\
2020\end{array}$ & $\begin{array}{l}\text { Random } \\
\text { cluster } \\
\text { sampling }\end{array}$ & 516 & $271(52.5)$ & Mean: 20.8 & $\begin{array}{l}\text { Online } \\
\text { self-administered } \\
\text { questionnaire }\end{array}$ & $\begin{array}{l}\text { Depression } \\
\text { subscale of } \\
\text { SCL-90-R } \geqslant 2\end{array}$ & $138(26.7)$ \\
\hline $\begin{array}{l}\text { Ma et al. } \\
(2020 b)\end{array}$ & $\begin{array}{l}\text { Undergraduates and } \\
\text { postgraduates of } 108 \\
\text { universities in } \\
\text { Guangdong and } \\
\text { Jiangxi, China }\end{array}$ & $\begin{array}{l}3-10 \text { February } \\
2020\end{array}$ & $\begin{array}{l}\text { Convenience } \\
\text { sampling }\end{array}$ & 746217 & $331613(44.4)$ & $\begin{array}{l}<18: 27640(3.7) \\
\text { 18-19: } 252616(33.9) \\
\text { 20-21: } 327639(43.9) \\
\text { 22-23: } 120142(16.1) \\
\text { 24-25: } 14925(2.0) \\
\geqslant 26: 3255(0.4)\end{array}$ & $\begin{array}{l}\text { Online } \\
\text { self-administered } \\
\text { questionnaire }\end{array}$ & $\mathrm{PHQ}-9 \geqslant 7$ & $\begin{array}{l}157452 \\
(21.1)\end{array}$ \\
\hline $\begin{array}{l}\text { Mao et al. } \\
(2020)\end{array}$ & $\begin{array}{l}\text { Medical postgraduates } \\
\text { of a general hospital in } \\
\text { Harbin, China }\end{array}$ & $\begin{array}{l}31 \text { March-10 May } \\
2020\end{array}$ & $\begin{array}{l}\text { Convenience } \\
\text { sampling }\end{array}$ & 240 & $124(51.7)$ & Mean: 24.3 & $\begin{array}{l}\text { Online } \\
\text { self-administered } \\
\text { questionnaire }\end{array}$ & $S D S \geqslant 50$ & $93(38.8)$ \\
\hline $\begin{array}{l}\text { Qian } \\
(2020)\end{array}$ & $\begin{array}{l}\text { Students of a medical } \\
\text { university in Fuzhou, } \\
\text { China }\end{array}$ & 10 March 2020 & $\begin{array}{l}\text { Convenience } \\
\text { sampling }\end{array}$ & 535 & $140(26.2)$ & Median: 21.0 & $\begin{array}{l}\text { Online } \\
\text { self-administered } \\
\text { questionnaire }\end{array}$ & $S D S \geqslant 50$ & $137(25.6)$ \\
\hline $\begin{array}{l}\text { Ren et al. } \\
(2020 a)\end{array}$ & $\begin{array}{l}\text { Undergraduates of a } \\
\text { university in } \\
\text { Zhongshan, China }\end{array}$ & NR & $\begin{array}{l}\text { Convenience } \\
\text { sampling }\end{array}$ & 244 & 85 (34.8) & NR & $\begin{array}{l}\text { Online } \\
\text { self-administered } \\
\text { questionnaire }\end{array}$ & $\begin{array}{l}\text { Depression } \\
\text { subscale of } \\
\text { DASS21 } \geqslant 10\end{array}$ & $78(32.0)$ \\
\hline $\begin{array}{l}\text { Ren et al. } \\
(2020 b)\end{array}$ & $\begin{array}{l}\text { Students of two } \\
\text { universities in Inner } \\
\text { Mongolia, China }\end{array}$ & $\begin{array}{l}21-28 \text { February } \\
2020\end{array}$ & $\begin{array}{l}\text { Convenience } \\
\text { sampling }\end{array}$ & 4560 & $1227(26.9)$ & Mean: 21.1 & $\begin{array}{l}\text { Online } \\
\text { self-administered } \\
\text { questionnaire }\end{array}$ & $S D S \geqslant 53$ & $3614(79.3)$ \\
\hline $\begin{array}{l}\text { Ren et al. } \\
(2020 c)\end{array}$ & $\begin{array}{l}\text { Nursing junior college } \\
\text { students and nursing } \\
\text { postgraduates of a } \\
\text { general hospital in } \\
\text { Shandong, China }\end{array}$ & 5-12 March 2020 & $\begin{array}{l}\text { Convenience } \\
\text { sampling }\end{array}$ & 294 & $64(21.8)$ & Mean: 21.6 & $\begin{array}{l}\text { Online } \\
\text { self-administered } \\
\text { questionnaire }\end{array}$ & $S D S \geqslant 50$ & $78(26.5)$ \\
\hline $\begin{array}{l}\text { Si et al. } \\
\text { (2020) }\end{array}$ & $\begin{array}{l}\text { Undergraduates and } \\
\text { postgraduates in seven } \\
\text { provinces in China }\end{array}$ & $\begin{array}{l}23 \text { February-5 } \\
\text { March } 2020\end{array}$ & $\begin{array}{l}\text { Convenience } \\
\text { sampling }\end{array}$ & 3606 & $1014(28.1)$ & $\begin{array}{l}\leqslant 20: 1467(40.7) \\
21-22: 1152(32.0) \\
\geqslant 23: 987(27.4)\end{array}$ & $\begin{array}{l}\text { Online } \\
\text { self-administered } \\
\text { questionnaire }\end{array}$ & $\begin{array}{l}\text { Depression } \\
\text { subscale of } \\
\text { DASS } 21 \geqslant 10\end{array}$ & $566(15.7)$ \\
\hline
\end{tabular}


Table 1. (Continued.)

\begin{tabular}{|c|c|c|c|c|c|c|c|c|c|}
\hline Study & Subjects and setting & $\begin{array}{l}\text { Dates of the } \\
\text { survey }\end{array}$ & $\begin{array}{l}\text { Sampling } \\
\text { method }\end{array}$ & Sample size & $\begin{array}{c}\text { Male students, } \\
n(\%)\end{array}$ & Age (years) & Survey method & $\begin{array}{l}\text { Assessment of } \\
\text { depressive } \\
\text { symptoms }\end{array}$ & $\begin{array}{l}\text { Depressed } \\
\text { students, } n \\
\text { (\%) }\end{array}$ \\
\hline $\begin{array}{l}\text { Sun et al. } \\
(2020)\end{array}$ & $\begin{array}{l}\text { Undergraduates in a } \\
\text { university in Hong } \\
\text { Kong, China }\end{array}$ & $\begin{array}{l}6 \text { June } 6-14 \text { July } \\
2020\end{array}$ & $\begin{array}{l}\text { Convenience } \\
\text { sampling }\end{array}$ & 255 & $33(12.9)$ & Mean: 21.0 & $\begin{array}{l}\text { Online } \\
\text { self-administered } \\
\text { questionnaire }\end{array}$ & CES-D- $10 \geqslant 10$ & $145(56.9)$ \\
\hline $\begin{array}{l}\text { Tang et al. } \\
(2020)\end{array}$ & $\begin{array}{l}\text { Undergraduates of six } \\
\text { universities in Chengdu } \\
\text { and Chongqing, China }\end{array}$ & $\begin{array}{l}20-27 \text { February } \\
2020\end{array}$ & $\begin{array}{l}\text { Convenience } \\
\text { sampling }\end{array}$ & 2485 & $960(38.6)$ & Mean: 19.8 & $\begin{array}{l}\text { Online } \\
\text { self-administered } \\
\text { questionnaire }\end{array}$ & PHQ-9 $\geqslant 10$ & $223(9.0)$ \\
\hline $\begin{array}{l}\text { Wan and } \\
\text { Shao } \\
(2020)\end{array}$ & $\begin{array}{l}\text { Students of three junior } \\
\text { colleges in } \\
\text { Heilongjiang, China }\end{array}$ & NR & $\begin{array}{l}\text { Convenience } \\
\text { sampling }\end{array}$ & 2358 & $1183(50.2)$ & NR & $\begin{array}{l}\text { Online } \\
\text { self-administered } \\
\text { questionnaire }\end{array}$ & PHQ-9 $\geqslant 5$ & $464(19.7)$ \\
\hline $\begin{array}{l}\text { Wang and } \\
\text { He (2020) }\end{array}$ & $\begin{array}{l}\text { University students in } \\
\text { Sichuan, Guizhou and } \\
\text { Chongqing, China }\end{array}$ & $\begin{array}{l}\text { Late February to } \\
\text { middle March, } \\
2020\end{array}$ & $\begin{array}{l}\text { Convenience } \\
\text { sampling }\end{array}$ & 1775 & NR $(<50)$ & NR & $\begin{array}{l}\text { Online } \\
\text { self-administered } \\
\text { questionnaire }\end{array}$ & $\begin{array}{l}\text { 'One SD above } \\
\text { the mean' on } \\
\text { depression } \\
\text { subscale of } \\
\text { PQEEPH }\end{array}$ & $308(17.4)$ \\
\hline $\begin{array}{l}\text { Wang and } \\
\text { Li (2020) }\end{array}$ & $\begin{array}{l}\text { Junior college students, } \\
\text { undergraduates and } \\
\text { postgraduates in } \\
\text { Sichuan, Yunnan and } \\
\text { Chongqing, China }\end{array}$ & February 2020 & $\begin{array}{l}\text { Convenience } \\
\text { sampling }\end{array}$ & 3178 & $878(27.6)$ & NR & $\begin{array}{l}\text { Online } \\
\text { self-administered } \\
\text { questionnaire }\end{array}$ & $\mathrm{SDS} \geqslant 50$ & $888(27.9)$ \\
\hline $\begin{array}{l}\text { Wang et al. } \\
(2020 b)\end{array}$ & $\begin{array}{l}\text { Students of a medical } \\
\text { university in Xi'an, } \\
\text { China }\end{array}$ & $\begin{array}{l}13-16 \text { February } \\
2020\end{array}$ & $\begin{array}{l}\text { Stratified } \\
\text { equal } \\
\text { proportion } \\
\text { sampling }\end{array}$ & 430 & $139(32.3)$ & Range: $18-25$ & $\begin{array}{l}\text { Online } \\
\text { self-administered } \\
\text { questionnaire }\end{array}$ & $\mathrm{SDS} \geqslant 53$ & $39(9.1)$ \\
\hline $\begin{array}{l}\text { Wang et al. } \\
(2020 c)\end{array}$ & Postgraduates in China & $\begin{array}{l}24 \text { February-7 } \\
\text { March } 2020\end{array}$ & $\begin{array}{l}\text { Convenience } \\
\text { sampling }\end{array}$ & 109 & $38(34.9)$ & $\begin{array}{l}20-25: 69.9 \\
26-30: 31.5 \\
31-40: 1.5\end{array}$ & $\begin{array}{l}\text { Online } \\
\text { self-administered } \\
\text { questionnaire }\end{array}$ & $S D S \geqslant 53$ & $22(20.2)$ \\
\hline $\begin{array}{l}\text { Wang et al. } \\
(2020 d)\end{array}$ & $\begin{array}{l}\text { Students of a university } \\
\text { in Hubei, China }\end{array}$ & 9-14 March 2020 & $\begin{array}{l}\text { Convenience } \\
\text { sampling }\end{array}$ & 2168 & $952(43.9)$ & Mean: 20.8 & $\begin{array}{l}\text { Online } \\
\text { self-administered } \\
\text { questionnaire }\end{array}$ & $C E S-D \geqslant 16$ & $752(34.7)$ \\
\hline $\begin{array}{l}\text { Wang et al. } \\
(2020 e)\end{array}$ & $\begin{array}{l}\text { Junior college students, } \\
\text { undergraduates and } \\
\text { postgraduates of } \\
\text { universities in Wuhan, } \\
\text { China }\end{array}$ & $\begin{array}{l}28 \text { May-3 June } \\
2020\end{array}$ & $\begin{array}{l}\text { Convenience } \\
\text { sampling }\end{array}$ & 3179 & $942(29.6)$ & NR & $\begin{array}{l}\text { Online } \\
\text { self-administered } \\
\text { questionnaire }\end{array}$ & PHQ-9 $\geqslant 5$ & $1123(35.3)$ \\
\hline $\begin{array}{l}\text { Wang et al. } \\
(2020 f)\end{array}$ & $\begin{array}{l}\text { Undergraduates and } \\
\text { postgraduates of four } \\
\text { universities in } \\
\text { Guangzhou, China }\end{array}$ & $\begin{array}{l}31 \text { January-5 } \\
\text { February } 2020\end{array}$ & $\begin{array}{l}\text { Cluster } \\
\text { sampling }\end{array}$ & 44447 & $20271(45.6)$ & Mean: 21.0 & $\begin{array}{l}\text { Online } \\
\text { self-administered } \\
\text { questionnaire }\end{array}$ & CES-D $\geqslant 28$ & $5404(12.2)$ \\
\hline Wei (2020) & $\begin{array}{l}\text { Students of a junior } \\
\text { college in Guangzhou, } \\
\text { China }\end{array}$ & $\begin{array}{l}13-18 \text { February } \\
2020\end{array}$ & $\begin{array}{l}\text { Convenience } \\
\text { sampling }\end{array}$ & 6289 & NR & NR & $\begin{array}{l}\text { Online } \\
\text { self-administered } \\
\text { questionnaire }\end{array}$ & PHQ-9 $\geqslant 5$ & $1310(20.8)$ \\
\hline $\begin{array}{l}\text { Wu et al. } \\
(2020)\end{array}$ & $\begin{array}{l}\text { Undergraduates of a } \\
\text { university in Shanghai, } \\
\text { China }\end{array}$ & March 2020 & $\begin{array}{l}\text { Random } \\
\text { sampling }\end{array}$ & 807 & $413(51.2)$ & NR & $\begin{array}{l}\text { Paper-pencil } \\
\text { self-administered } \\
\text { questionnaire }\end{array}$ & $\begin{array}{l}\text { Depression } \\
\text { subscale of } \\
S C L-90-R \geqslant 2\end{array}$ & $216(26.8)$ \\
\hline
\end{tabular}




\begin{tabular}{|c|c|c|c|c|c|c|c|c|c|}
\hline $\begin{array}{l}\text { Xiang et al. } \\
(2020)\end{array}$ & $\begin{array}{l}\text { Undergraduates and } \\
\text { postgraduates in China }\end{array}$ & $\begin{array}{l}25 \text { February-25 } \\
\text { March } 2020\end{array}$ & $\begin{array}{l}\text { Convenience } \\
\text { sampling }\end{array}$ & 1396 & 881 (63.1) & Mean: 20.7 & $\begin{array}{l}\text { Online } \\
\text { self-administered } \\
\text { questionnaire }\end{array}$ & SDS $>50$ & $583(41.8)$ \\
\hline $\begin{array}{l}\text { Xiao et al. } \\
(2020 a)\end{array}$ & $\begin{array}{l}\text { Undergraduates and } \\
\text { postgraduates of two } \\
\text { medical universities in } \\
\text { Beijing and Wuhan, } \\
\text { China }\end{array}$ & $\begin{array}{l}\text { 4-12 February } \\
2020\end{array}$ & $\begin{array}{l}\text { Cluster } \\
\text { sampling }\end{array}$ & $\begin{array}{l}933 \text { (Beijing: } \\
\text { 558; Wuhan: } \\
\text { 375) }\end{array}$ & 279 (29.9) & $\begin{array}{l}\text { 17-24: } 755(80.9) \\
\text { >25: } \\
178(19.1)\end{array}$ & $\begin{array}{l}\text { Online } \\
\text { self-administered } \\
\text { questionnaire }\end{array}$ & PHQ-9 $\geqslant 5$ & $\begin{array}{l}236(25.3) \\
\text { Beijing: } 131 \\
\text { (23.5) } \\
\text { Wuhan: } 105 \\
\text { (28.0) }\end{array}$ \\
\hline $\begin{array}{l}\text { Xiao et al. } \\
(2020 b)\end{array}$ & $\begin{array}{l}\text { Undergraduates of two } \\
\text { universities in Wuhan, } \\
\text { China }\end{array}$ & $\begin{array}{l}\text { 5-9 February } \\
2020\end{array}$ & $\begin{array}{l}\text { Stratified } \\
\text { cluster } \\
\text { sampling }\end{array}$ & 3966 & $1591(40.1)$ & NR & $\begin{array}{l}\text { Online } \\
\text { self-administered } \\
\text { questionnaire }\end{array}$ & PHQ-9 $\geqslant 5$ & $1075(27.1)$ \\
\hline $\begin{array}{l}\text { Xie et al. } \\
(2020)\end{array}$ & $\begin{array}{l}\text { Undergraduates of } \\
\text { provinces other than } \\
\text { Hubei in China }\end{array}$ & $\begin{array}{l}4-7 \text { February } \\
2020\end{array}$ & $\begin{array}{l}\text { Convenience } \\
\text { sampling }\end{array}$ & 2705 & $608(22.5)$ & NR & $\begin{array}{l}\text { Online } \\
\text { self-administered } \\
\text { questionnaire }\end{array}$ & PHQ-9 $\geqslant 5$ & $493(18.2)$ \\
\hline $\begin{array}{l}\text { Xin et al. } \\
(2020)\end{array}$ & $\begin{array}{l}\text { Undergraduates and } \\
\text { postgraduates in China }\end{array}$ & $\begin{array}{l}\text { 1-10 February } \\
2020\end{array}$ & $\begin{array}{l}\text { Stratified } \\
\text { cluster } \\
\text { sampling }\end{array}$ & 24378 & 7865 (32.3) & Mean: 19.9 & $\begin{array}{l}\text { Online } \\
\text { self-administered } \\
\text { questionnaire }\end{array}$ & PHQ- $9 \geqslant 10$ & 3619 (14.8) \\
\hline $\begin{array}{l}\text { Xing et al. } \\
(2020)\end{array}$ & $\begin{array}{l}\text { Medical } \\
\text { undergraduates of two } \\
\text { universities in } \\
\text { Hangzhou, China }\end{array}$ & $\begin{array}{l}5-7 \text { February } \\
2020\end{array}$ & $\begin{array}{l}\text { Convenience } \\
\text { sampling }\end{array}$ & 595 & $174(29.2)$ & NR & $\begin{array}{l}\text { Online } \\
\text { self-administered } \\
\text { questionnaire }\end{array}$ & PHQ-9 $\geqslant 5$ & $114(19.2)$ \\
\hline $\begin{array}{l}\text { Xiong } \\
\text { et al. } \\
\text { (2020) }\end{array}$ & $\begin{array}{l}\text { Undergraduates and } \\
\text { postgraduates of a } \\
\text { university in } \\
\text { Guangzhou, China }\end{array}$ & $\begin{array}{l}20 \text { February-20 } \\
\text { March } 2020\end{array}$ & $\begin{array}{l}\text { Convenience } \\
\text { sampling }\end{array}$ & 563 & $172(30.6)$ & Mean: 21.5 & $\begin{array}{l}\text { Online } \\
\text { self-administered } \\
\text { questionnaire }\end{array}$ & $\begin{array}{l}\text { Depression } \\
\text { subscale of } \\
\text { DASS21 } \geqslant 14\end{array}$ & $69(12.3)$ \\
\hline $\begin{array}{l}\mathrm{Xu} \text { and } \mathrm{Li} \\
(2020)\end{array}$ & $\begin{array}{l}\text { Undergraduates of a } \\
\text { university in Hubei, } \\
\text { China }\end{array}$ & 18-31 May 2020 & $\begin{array}{l}\text { Cluster } \\
\text { sampling }\end{array}$ & 6891 & $2113(30.7)$ & NR & $\begin{array}{l}\text { Online } \\
\text { self-administered } \\
\text { questionnaire }\end{array}$ & $S D S \geqslant 53$ & $1874(27.2)$ \\
\hline $\begin{array}{l}\text { Yan et al. } \\
(2020)\end{array}$ & $\begin{array}{l}\text { Medical } \\
\text { undergraduates in } \\
\text { Putian, China }\end{array}$ & $\begin{array}{l}23 \text { January-23 } \\
\text { February } 2020\end{array}$ & $\begin{array}{l}\text { Cluster } \\
\text { sampling }\end{array}$ & 634 & $89(14.0)$ & Mean: 19.3 & $\begin{array}{l}\text { Online } \\
\text { self-administered } \\
\text { questionnaire }\end{array}$ & $\begin{array}{l}\text { Depression } \\
\text { subscale of } \\
\text { HADS }>7\end{array}$ & $146(23.0)$ \\
\hline $\begin{array}{l}\text { Yang et al. } \\
(2020 b)\end{array}$ & $\begin{array}{l}\text { Undergraduates and } \\
\text { postgraduates of } \\
\text { Universities in Shaanxi, } \\
\text { China }\end{array}$ & $\begin{array}{l}7-9 \text { February } \\
2020\end{array}$ & $\begin{array}{l}\text { Convenience } \\
\text { sampling }\end{array}$ & 1667 & $803(48.2)$ & Mean: 20.6 & $\begin{array}{l}\text { Online } \\
\text { self-administered } \\
\text { questionnaire }\end{array}$ & $\begin{array}{l}\text { 'One SD above } \\
\text { the mean' on } \\
\text { depression } \\
\text { subscale of } \\
\text { PQEEPH }\end{array}$ & $257(15.4)$ \\
\hline $\begin{array}{l}\text { Yao et al. } \\
(2020)\end{array}$ & $\begin{array}{l}\text { Students of a military } \\
\text { university in China }\end{array}$ & $\begin{array}{l}27-28 \text { February } \\
2020\end{array}$ & $\begin{array}{l}\text { Convenience } \\
\text { sampling }\end{array}$ & 84 & $52(61.9)$ & Mean: 19.9 & $\begin{array}{l}\text { Online } \\
\text { self-administered } \\
\text { questionnaire }\end{array}$ & PHQ-9 $\geqslant 5$ & $21(25.0)$ \\
\hline $\begin{array}{l}\text { Yi et al. } \\
(2020 a)\end{array}$ & $\begin{array}{l}\text { Undergraduates of a } \\
\text { university in Zhanjiang, } \\
\text { China }\end{array}$ & 2-8 March 2020 & $\begin{array}{l}\text { Cluster } \\
\text { sampling }\end{array}$ & 393 & $121(30.8)$ & Mean: 21.7 & $\begin{array}{l}\text { Online } \\
\text { self-administered } \\
\text { questionnaire }\end{array}$ & $S D S \geqslant 53$ & $104(26.5)$ \\
\hline $\begin{array}{l}\text { Yi et al. } \\
(2020 b)\end{array}$ & $\begin{array}{l}\text { Undergraduates of a } \\
\text { medical college in } \\
\text { Xinxiang, China }\end{array}$ & $\begin{array}{l}22-24 \text { February } \\
2020\end{array}$ & $\begin{array}{l}\text { Convenience } \\
\text { sampling }\end{array}$ & 1234 & $462(37.4)$ & NR & $\begin{array}{l}\text { Online } \\
\text { self-administered } \\
\text { questionnaire }\end{array}$ & PHQ-9 $\geqslant 5$ & $276(22.4)$ \\
\hline $\begin{array}{l}\text { Yu et al. } \\
\text { (2020) }\end{array}$ & $\begin{array}{l}\text { Undergraduates in } \\
\text { Guangdong, China }\end{array}$ & NR & $\begin{array}{l}\text { Convenience } \\
\text { sampling }\end{array}$ & 427 & $98(23.0)$ & NR & $\begin{array}{l}\text { Online } \\
\text { self-administered } \\
\text { questionnaire }\end{array}$ & $S D S \geqslant 53$ & $129(30.2)$ \\
\hline
\end{tabular}


Table 1. (Continued.)

\begin{tabular}{|c|c|c|c|c|c|c|c|c|c|}
\hline Study & Subjects and setting & $\begin{array}{l}\text { Dates of the } \\
\text { survey }\end{array}$ & $\begin{array}{l}\text { Sampling } \\
\text { method }\end{array}$ & Sample size & $\begin{array}{c}\text { Male students, } \\
n(\%)\end{array}$ & Age (years) & Survey method & $\begin{array}{l}\text { Assessment of } \\
\text { depressive } \\
\text { symptoms }\end{array}$ & $\begin{array}{l}\text { Depressed } \\
\text { students, } n \\
\quad(\%)\end{array}$ \\
\hline $\begin{array}{l}\text { Zhan et al. } \\
(2020)\end{array}$ & $\begin{array}{l}\text { Junior college students, } \\
\text { undergraduates and } \\
\text { postgraduates of four } \\
\text { medical universities in } \\
\text { Hunan and Fujian, } \\
\text { China }\end{array}$ & $\begin{array}{l}17-19 \text { March } \\
2020\end{array}$ & $\begin{array}{l}\text { Convenience } \\
\text { sampling }\end{array}$ & 266 & $76(28.6)$ & $\begin{array}{l}<18: 16(6.0) \\
\text { 18-25: } 188(70.7) \\
26-35: 58(21.8) \\
>35: 4(1.5)\end{array}$ & $\begin{array}{l}\text { Online } \\
\text { self-administered } \\
\text { questionnaire }\end{array}$ & $\begin{array}{l}\text { Depression } \\
\text { subscale of } \\
\text { DASS21 } 210\end{array}$ & $54(20.3)$ \\
\hline $\begin{array}{l}\text { Zhang } \\
\text { et al. } \\
(2020 b)\end{array}$ & $\begin{array}{l}\text { Undergraduates of four } \\
\text { universities in } \\
\text { Guangdong, China }\end{array}$ & $\begin{array}{l}31 \text { January-4 } \\
\text { February } 2020\end{array}$ & $\begin{array}{l}\text { Convenience } \\
\text { sampling }\end{array}$ & 312 & $67(21.5)$ & Mean: 19.6 & $\begin{array}{l}\text { Online } \\
\text { self-administered } \\
\text { questionnaire }\end{array}$ & PHQ-9 $\geqslant 5$ & $92(29.5)$ \\
\hline $\begin{array}{l}\text { Zhang } \\
\text { et al. } \\
\text { (2020c) }\end{array}$ & $\begin{array}{l}\text { University students in } \\
\text { China }\end{array}$ & $\begin{array}{l}4-7 \text { February } \\
2020\end{array}$ & $\begin{array}{l}\text { Convenience } \\
\text { sampling }\end{array}$ & 7833 & $2081(26.6)$ & Mean: 19.8 & $\begin{array}{l}\text { Online } \\
\text { self-administered } \\
\text { questionnaire }\end{array}$ & $\mathrm{PHQ}-9 \geqslant 5$ & $3053(39.0)$ \\
\hline $\begin{array}{l}\text { Yang et al. } \\
(2020 a) \text {, } \\
\text { Zhang } \\
\text { et al. } \\
(2020 d)\end{array}$ & $\begin{array}{l}\text { Medical } \\
\text { undergraduates in } \\
\text { China }\end{array}$ & $\begin{array}{l}11-19 \text { February } \\
2020\end{array}$ & $\begin{array}{l}\text { Convenience } \\
\text { sampling }\end{array}$ & 6226 & 2484 (39.9) & Range: $18-27$ & $\begin{array}{l}\text { Online } \\
\text { self-administered } \\
\text { questionnaire }\end{array}$ & PHQ-9 $\geqslant 5$ & $2206(35.4)$ \\
\hline $\begin{array}{l}\text { Zhang } \\
\text { et al. } \\
\text { (2020e) }\end{array}$ & $\begin{array}{l}\text { Medical } \\
\text { undergraduates of a } \\
\text { university in Chenzhou, } \\
\text { China }\end{array}$ & $\begin{array}{l}27-29 \text { February } \\
2020\end{array}$ & $\begin{array}{l}\text { Cluster } \\
\text { sampling }\end{array}$ & 932 & $505(54.2)$ & NR & $\begin{array}{l}\text { Online } \\
\text { self-administered } \\
\text { questionnaire }\end{array}$ & PHQ-9 $\geqslant 5$ & $270(29.0)$ \\
\hline $\begin{array}{l}\text { Zhang } \\
\text { et al. } \\
\text { (2020f) }\end{array}$ & $\begin{array}{l}\text { Medical students of two } \\
\text { universities in Inner } \\
\text { Mongolia, China }\end{array}$ & February 2020 & $\begin{array}{l}\text { Random } \\
\text { sampling }\end{array}$ & 1486 & $453(30.5)$ & Mean: 21.7 & $\begin{array}{l}\text { Online } \\
\text { self-administered } \\
\text { questionnaire }\end{array}$ & PHQ-9 $\geqslant 5$ & $528(35.5)$ \\
\hline $\begin{array}{l}\text { Zhang } \\
\text { et al. } \\
(2020 \mathrm{~g})\end{array}$ & $\begin{array}{l}\text { Undergraduates and } \\
\text { postgraduates in China }\end{array}$ & $\begin{array}{l}\text { February-April, } \\
2020\end{array}$ & $\begin{array}{l}\text { Convenience } \\
\text { sampling }\end{array}$ & 1409 & $733(52.0)$ & NR & $\begin{array}{l}\text { Online } \\
\text { self-administered } \\
\text { questionnaire }\end{array}$ & $S D S \geqslant 53$ & $160(11.4)$ \\
\hline $\begin{array}{l}\text { Zhang } \\
\text { et al. } \\
(2020 h)\end{array}$ & $\begin{array}{l}\text { Students of } 57 \\
\text { universities in China }\end{array}$ & $\begin{array}{l}21-24 \text { February } \\
2020\end{array}$ & $\begin{array}{l}\text { Convenience } \\
\text { sampling }\end{array}$ & 2270 & $877(38.6)$ & $\begin{array}{l}\leqslant 19: 660(29.1) \\
20-23: 1458(64.2) \\
\geqslant 24: 152(6.7)\end{array}$ & $\begin{array}{l}\text { Online } \\
\text { self-administered } \\
\text { questionnaire }\end{array}$ & SDS $>53$ & $237(10.4)$ \\
\hline $\begin{array}{l}\text { Zhao et al. } \\
(2020 a)\end{array}$ & $\begin{array}{l}\text { Undergraduates and } \\
\text { postgraduates in China }\end{array}$ & $\begin{array}{l}23 \text { March-20 } \\
\text { April } 2020\end{array}$ & $\begin{array}{l}\text { Convenience } \\
\text { sampling }\end{array}$ & 281 & $83(29.5)$ & Mean: 23.6 & $\begin{array}{l}\text { Online } \\
\text { self-administered } \\
\text { questionnaire }\end{array}$ & $\mathrm{PHQ}-9 \geqslant 5$ & $170(60.5)$ \\
\hline $\begin{array}{l}\text { Zhao et al. } \\
(2020 b)\end{array}$ & $\begin{array}{l}\text { Chinese } \\
\text { undergraduates and } \\
\text { postgraduates in South } \\
\text { Korea }\end{array}$ & $\begin{array}{l}23 \text { March-8 } \\
\text { April } 2020\end{array}$ & $\begin{array}{l}\text { Convenience } \\
\text { sampling }\end{array}$ & 171 & $57(33.3)$ & Mean: 24.1 & $\begin{array}{l}\text { Online } \\
\text { self-administered } \\
\text { questionnaire }\end{array}$ & PHQ-9 $\geqslant 10$ & $49(28.7)$ \\
\hline $\begin{array}{l}\text { Zhao et al. } \\
\text { (2020c) }\end{array}$ & $\begin{array}{l}\text { University students in } \\
\text { China }\end{array}$ & NR & $\begin{array}{l}\text { Convenience } \\
\text { sampling }\end{array}$ & 364 & NR & NR & $\begin{array}{l}\text { Online } \\
\text { self-administered } \\
\text { questionnaire }\end{array}$ & $\begin{array}{l}\text { Depression } \\
\text { subscale of } \\
\text { DASS21 } \geqslant 10\end{array}$ & $118(32.4)$ \\
\hline $\begin{array}{l}\text { Zhao and } \\
\text { Hu (2020) }\end{array}$ & $\begin{array}{l}\text { Undergraduates of a } \\
\text { medical college in } \\
\text { Ganzhou, China }\end{array}$ & March-April 2020 & $\begin{array}{l}\text { Convenience } \\
\text { sampling }\end{array}$ & 456 & $240(52.6)$ & Mean: 22.1 & $\begin{array}{l}\text { Online } \\
\text { self-administered } \\
\text { questionnaire }\end{array}$ & $\mathrm{SDS} \geqslant 53$ & $50(11.0)$ \\
\hline
\end{tabular}




\begin{tabular}{|c|c|c|c|c|c|c|c|c|c|}
\hline $\begin{array}{l}\text { Zhou et al. } \\
\text { (2020) }\end{array}$ & $\begin{array}{l}\text { Undergraduates and } \\
\text { postgraduates in China }\end{array}$ & 1-15 March 2020 & $\begin{array}{l}\text { Convenience } \\
\text { sampling }\end{array}$ & 11133 & 4195 (37.7) & Median: 21.0 & $\begin{array}{l}\text { Online } \\
\text { self-administered } \\
\text { questionnaire }\end{array}$ & PHQ-9 $\geqslant 5$ & $4119(37.0)$ \\
\hline $\begin{array}{l}\text { Chen and } \\
\text { Zhu (2021) }\end{array}$ & $\begin{array}{l}\text { Undergraduates and } \\
\text { postgraduates of a } \\
\text { university in Shanghai, } \\
\text { China }\end{array}$ & $\begin{array}{l}12-15 \text { March } \\
2020\end{array}$ & $\begin{array}{l}\text { Convenience } \\
\text { sampling }\end{array}$ & 3353 & $1651(49.2)$ & Mean: 21.8 & $\begin{array}{l}\text { Online } \\
\text { self-administered } \\
\text { questionnaire }\end{array}$ & CES-D- $11 \geqslant 10$ & $1693(50.5)$ \\
\hline $\begin{array}{l}\text { Ni et al. } \\
(2021)\end{array}$ & $\begin{array}{l}\text { Medical postgraduates } \\
\text { of a general hospital in } \\
\text { Nanjing, China }\end{array}$ & 4 March 2020 & $\begin{array}{l}\text { Convenience } \\
\text { sampling }\end{array}$ & 157 & NR & NR & $\begin{array}{l}\text { Online } \\
\text { self-administered } \\
\text { questionnaire }\end{array}$ & $S D S \geqslant 53$ & $76(48.4)$ \\
\hline $\begin{array}{l}\text { Pan et al. } \\
\text { (2021) }\end{array}$ & $\begin{array}{l}\text { Undergraduates and } \\
\text { postgraduates in China }\end{array}$ & 4-9 March 2020 & $\begin{array}{l}\text { Convenience } \\
\text { sampling }\end{array}$ & 3975 & $1611(40.5)$ & Range: $16-30$ & $\begin{array}{l}\text { Online } \\
\text { self-administered } \\
\text { questionnaire }\end{array}$ & $C E S-D \geqslant 16$ & $1568(39.4)$ \\
\hline $\begin{array}{l}\text { Sun et al. } \\
(2021)\end{array}$ & $\begin{array}{l}\text { Undergraduates and } \\
\text { postgraduates in China }\end{array}$ & $\begin{array}{l}20 \text { March-April } \\
2020\end{array}$ & $\begin{array}{l}\text { Convenience } \\
\text { sampling }\end{array}$ & 1912 & $578(30.2)$ & Mean: 20.3 & $\begin{array}{l}\text { Online } \\
\text { self-administered } \\
\text { questionnaire }\end{array}$ & $\mathrm{PHQ}-9 \geqslant 5$ & $890(46.5)$ \\
\hline $\begin{array}{l}\text { Wang et al. } \\
\text { (2021) }\end{array}$ & $\begin{array}{l}\text { Junior college students, } \\
\text { undergraduates and } \\
\text { postgraduates in Anhui, } \\
\text { China }\end{array}$ & $\begin{array}{l}18-20 \text { February } \\
2020\end{array}$ & $\begin{array}{l}\text { Convenience } \\
\text { sampling }\end{array}$ & 840 & 276 (32.9) & Mean: 20.2 & $\begin{array}{l}\text { Online } \\
\text { self-administered } \\
\text { questionnaire }\end{array}$ & $S D S \geqslant 53$ & $233(27.7)$ \\
\hline $\begin{array}{l}\text { Wu et al. } \\
(2021)\end{array}$ & $\begin{array}{l}\text { Undergraduates in } 16 \\
\text { provinces and cities in } \\
\text { China }\end{array}$ & $\begin{array}{l}4-12 \text { February } \\
2020\end{array}$ & $\begin{array}{l}\text { Random } \\
\text { sampling }\end{array}$ & 11787 & $5056(42.9)$ & Mean: 20.5 & $\begin{array}{l}\text { Online } \\
\text { self-administered } \\
\text { questionnaire }\end{array}$ & $\mathrm{PHQ}-9 \geqslant 5$ & 3053 (25.9) \\
\hline $\begin{array}{l}\text { Yu et al. } \\
(2021)\end{array}$ & $\begin{array}{l}\text { Undergraduates in } \\
\text { China }\end{array}$ & 3-15 March 2020 & $\begin{array}{l}\text { Convenience } \\
\text { sampling }\end{array}$ & 1681 & $592(35.2)$ & NR & $\begin{array}{l}\text { Online } \\
\text { self-administered } \\
\text { questionnaire }\end{array}$ & $C E S-D \geqslant 16$ & $955(56.8)$ \\
\hline
\end{tabular}

NR, not reported; s.D., standard deviation; PHQ-9, 9-item Patient Health Questionnaire; DASS-21, Depression, Anxiety and Stress Scale - 21 Items; PQEEPH, Psychological Questionnaires for Emergent Events of Public Health; SCL-90-R, Symptom Checklist-90-Revised; CES-D, Center for Epidemiologic Studies Depression Scale; SDS, Zung's Self-Depression Rating Scale; HADS, Hospital Anxiety and Depression Scale. 
Study

Cao, 2020

Chang et al., 2020

Chen et al., 2020a

Chen et al., 2020b

Chen et al., 2020c

Chen et al., 2020d

Chi et al., 2020

Cong et al., 2020

Deng et al., 2020

Dong et al., 2020

Dong, 2020

Feng et al., 2020

Feng, 2020

Han et al., 2020

Ji et al., 2020

Jiang et al., 2020

Lei et al., 2020

$\mathrm{Li}$ and $\mathrm{He}, 2020$

Li et al., 2020b

Lian et al., 2020

Liang et al., 2020a

Liang et al., 2020b

Lin et al., 2020a

Lin et al., 2020b

Lin and Xu, 2020

Liu et al., 2020a

Liu et al., 2020b

Liu et al., 2020c

Liu, 2020a

Liu, 2020b

Ma et al., 2020a

Ma et al., 2020b

Mao et al., 2020

Qian, 2020

Ren et al., 2020a

Ren et al., 2020b

Ren et al., 2020c

Si et al., 2020

Sun et al., 2020

Tang et al., 2020

Wan and Shao, 2020

Wang and $\mathrm{He}, 2020$

Wang and $\mathrm{Li}, 2020$

Wang et al., 2020b

Wang et al., 2020c

Wang et al., 2020d

Wang et al., 2020

Wang et al., 2020f

Wei, 2020

Wu et al., 2020

Xiang et al., 2020

Xiao et al., 2020a

Xiao et al., 2020b

Xie et al., 2020

Xin et al., 2020

Xing et al., 2020

Xiong et al., 2020

$\mathrm{Xu}$ and $\mathrm{Li}, 2020$

Yan et al., 2020

Yang et al., 2020b

Yao et al., 2020

Yi et al., 2020a

Yi et al., 2020b

Yu et al., 2020

Zhan et al., 2020

Zhang et al., 2020b

Zhang et al., 2020c

Zhang et al., 2020d

Zhang et al., 2020e

Zhang et al., $2020 \mathrm{f}$

Zhang et al., 2020g

Zhang et al., 2020h

Zhao et al., 2020a

Zhao et al., 2020b

Zhao et al., 2020c

Zhao and Hu, 2020

Zhou et al., 2020

Chen and Zhu, 2021

$\mathrm{Ni}$ et al., 2021

Pan et al., 2021

Sun et al., 202

Wang et al., 2021

Wu et al., 2021

Yu et al., 2021

Events

$\begin{array}{rr}575 & 27 \\ 821 & 38 \\ 172 & 79 \\ 24909 & 323489\end{array}$

$\begin{array}{rr}54 & 2 \\ 61 & 6 \\ 475 & 2038\end{array}$

$152 \quad 25$

$56 \quad 1607$

$554 \quad 4085$

$429 \quad 134$

$1956 \quad 7157$

$178 \quad 405$

$247 \quad 1013$

$\begin{array}{ll}104 & 399 \\ 143 & 231\end{array}$

$240 \quad 1144$

$1278 \quad 7747$

177

15
40

$217 \quad 625$

$183 \quad 320$

$320 \quad 1297$

77

101

70

14
29

138

157452

$\begin{array}{lr}93 & 240\end{array}$

$137 \quad 53$

$78 \quad 244$

$3614 \quad 4560$

$78 \quad 294$

$\begin{array}{rr}566 & 360 \\ 145 & 255\end{array}$

$223 \quad 2485$

$464 \quad 235$

$308 \quad 177$

$888 \quad 3178$

$\begin{array}{ll}39 & 430 \\ 22 & 109\end{array}$

$\begin{array}{rr}22 & 109 \\ 752 & 2168\end{array}$

$1123 \quad 3179$

$5404 \quad 44447$

$1310 \quad 6289$

$216 \quad 807$

$583 \quad 1396$

$236 \quad 933$

10753966

$493 \quad 2705$

$3619 \quad 24378$

$114 \quad 595$

$69 \quad 563$

$1874 \quad 6891$

$146 \quad 634$

$257 \quad 1667$

$\begin{array}{rr}21 & 84 \\ 104 & 393\end{array}$

$104 \quad 393$

$276 \quad 1234$

$\begin{array}{rr}129 & 427 \\ 54 & 266\end{array}$

$92 \quad 312$

$3053 \quad 7833$

$2206 \quad 6226$

$270 \quad 932$

$528 \quad 1486$

$160 \quad 1409$

$237 \quad 2270$

$170 \quad 281$

$49 \quad 171$

$118 \quad 364$

$50 \quad 456$

$4119 \quad 11133$

$1693 \quad 3353$

$76 \quad 157$

$1568 \quad 3975$

$890 \quad 1912$

$233 \quad 840$

$\begin{array}{rr}3053 & 11787 \\ 955 & 1681\end{array}$

Random effects model 1292811 . 2. Forest plot of prevalence of depressive symptoms pandemic.
Heterogeneity: $I^{2}=99.9 \%, \tau^{2}=0.0197, p=0$

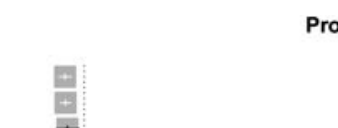

Proportion

$95 \%-\mathrm{Cl}$ Weight

$0.2104[0.1952 ; 0.2262] \quad 1.2 \%$

$0.2115[0.1988 ; 0.2247] \quad 1.2 \%$

$0.2164[0.1882 ; 0.2466] \quad 1.2 \%$

$0.0770[0.0761 ; 0.0779] \quad 1.2 \%$

$0.1888[0.1451 ; 0.2391]-12 \%$

$0.0875[0.0676 ; 0.1110] \quad 1.2 \%$

$0.2331[0.2149 ; 0.2520] \quad 1.2 \%$

$0.6032[0.5399 ; 0.6640] \quad 1.2 \%$

$0.0348[0.0264 ; 0.0450] \quad 1.2 \%$

$0.3889[0.3134 ; 0.4685] \quad 1.1 \%$

$0.1356[0.1253 ; 0.1465] \quad 1.2 \%$

$0.3187[0.2939 ; 0.3444] \quad 1.2 \%$

$0.2733[0.2630 ; 0.2838] \quad 1.2 \%$

$0.4395[0.3905 ; 0.4894] \quad 1.2 \%$

$0.2438[0.2177 ; 0.2715] \quad 1.2 \%$

$0.2607[0.2182 ; 0.3067] \quad 1.2 \%$

$0.6190[0.5531 ; 0.6819] \quad 1.2 \%$

$0.2098[0.1865 ; 0.2346] \quad 1.2 \%$

$0.1650[0.1568 ; 0.1734] \quad 1.2 \%$

$0.1232[0.1066 ; 0.1413] \quad 1.2 \%$

$0.0176[0.0099 ; 0.0289] \quad 1.2 \%$

$0.0504[0.0363 ; 0.0681] \quad 1.2 \%$

0.3472 [0.3099; 0.3860$] \quad 1.2 \%$

$0.5719[0.5156 ; 0.6268] \quad 1.2 \%$

$0.2467[0.2235 ; 0.2711] \quad 1.2 \%$

$0.3548[0.2913 ; 0.4224] \quad 1.2 \%$

$0.1653[0.1367 ; 0.1972] \quad 1.2 \%$

$0.1375[0.1088 ; 0.1705] \quad 1.2 \%$

$0.0733[0.0407 ; 0.1199] \quad 1.1 \%$

$0.3222[0.2275 ; 0.4290] \quad 1.1 \%$

$0.2674[0.2297 ; 0.3079] \quad 1.2 \%$

$0.2110[0.2101 ; 0.2119] \quad 1.2 \%$

$0.3875[0.3255 ; 0.4523] \quad 1.2 \%$

$0.2561[0.2196 ; 0.2953] \quad 1.2 \%$

$0.3197[0.2616 ; 0.3822] \quad 1.2 \%$

$0.7925[0.7805 ; 0.8042] \quad 1.2 \%$

$0.2653[0.2157 ; 0.3197] \quad 1.2 \%$

$0.1570[0.1452 ; 0.1692] \quad 1.2 \%$

$0.5686[0.5054 ; 0.6303] \quad 1.2 \%$

$0.0897[0.0788 ; 0.1017] \quad 1.2 \%$

$0.1968[0.1809 ; 0.2134] \quad 1.2 \%$

$0.1735[0.1562 ; 0.1920] \quad 1.2 \%$

$0.2794[0.2639 ; 0.2954] \quad 1.2 \%$

$0.0907[0.0653 ; 0.1219] \quad 1.2 \%$

$0.2018[0.1310 ; 0.2895] \quad 1.1 \%$

0.3469 [0.3268; 0.3673$] \quad 1.2 \%$

$0.3533[0.3366 ; 0.3702] \quad 1.2 \%$

$0.1216[0.1186 ; 0.1247] \quad 1.2 \%$

$0.2083[0.1983 ; 0.2186] \quad 1.2 \%$

$0.2677[0.2374 ; 0.2996] \quad 1.2 \%$

$0.4176[0.3916 ; 0.4440] \quad 1.2 \%$

$0.2529[0.2253 ; 0.2821] \quad 1.2 \%$

$0.2711[0.2573 ; 0.2852] \quad 1.2 \%$

$0.1823[0.1679 ; 0.1973] \quad 1.2 \%$

$0.1485[0.1440 ; 0.1530] \quad 1.2 \%$

$0.1916[0.1607 ; 0.2256] \quad 1.2 \%$

$0.1226[0.0966 ; 0.1525] \quad 1.2 \%$

$0.2719[0.2615 ; 0.2826] \quad 1.2 \%$

$0.2303[0.1980 ; 0.2651] \quad 1.2 \%$

$0.1542[0.1372 ; 0.1724] \quad 1.2 \%$

$0.2500[0.1619 ; 0.3564] \quad 1.1 \%$

$0.2646[0.2217 ; 0.3112] \quad 1.2 \%$

$0.2237[0.2007 ; 0.2480] \quad 1.2 \%$

$0.3021[0.2589 ; 0.3481] \quad 1.2 \%$

$0.2030[0.1563 ; 0.2564] \quad 1.2 \%$

0.2949 [0.2448; 0.3489$] \quad 1.2 \%$

$0.3898[0.3789 ; 0.4007] \quad 1.2 \%$

$0.3543[0.3424 ; 0.3663] \quad 1.2 \%$

$0.2897[0.2607 ; 0.3200] 1.2 \%$

$0.3553[0.3310 ; 0.3803] \quad 1.2 \%$

$0.1136[0.0975 ; 0.1313] \quad 1.2 \%$

$0.1044[0.0921 ; 0.1177] \quad 1.2 \%$

$0.6050[0.5452 ; 0.6625] \quad 1.2 \%$

$0.2865[0.2201 ; 0.3606] \quad 1.1 \%$

$0.3242[0.2763 ; 0.3749] \quad 1.2 \%$

$0.1096[0.0825 ; 0.1420] \quad 1.2 \%$

$0.3700[0.3610 ; 0.3790] \quad 1.2 \%$

$0.5049[0.4879 ; 0.5220] \quad 1.2 \%$

$0.4841[0.4037 ; 0.5651] \quad 1.1 \%$

$0.3945[0.3792 ; 0.4099] \quad 1.2 \%$

0.4655 [0.4429; 0.4881$] \quad 1.2 \%$

$0.2774[0.2473 ; 0.3090] \quad 1.2 \%$

$0.2590[0.2511 ; 0.2670] \quad 1.2 \%$

0.5681 [ $0.5440 ; 0.5920] \quad 1.2 \%$

$0.2597[0.2334 ; 0.2868] 100.0 \%$ 


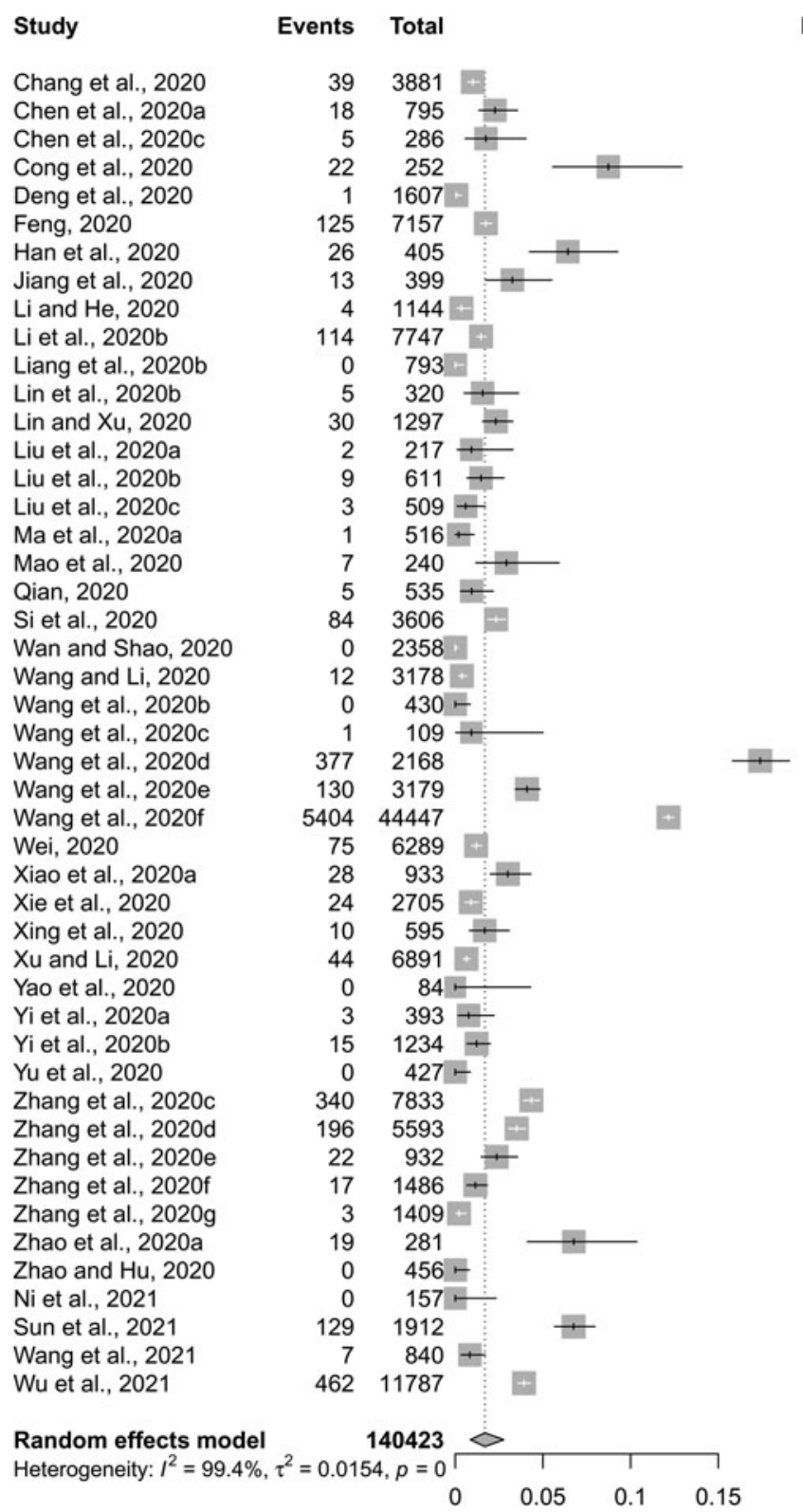

\begin{tabular}{ll} 
Proportion & 95\%-Cl Weight \\
& \\
$0.0100[0.0072 ; 0.0137]$ & $2.2 \%$ \\
$0.0226[0.0135 ; 0.0355]$ & $2.1 \%$ \\
$0.0175[0.0057 ; 0.0403]$ & $2.1 \%$ \\
$0.0873[0.0555 ; 0.1292]$ & $2.1 \%$ \\
$0.0006[0.0000 ; 0.0035]$ & $2.2 \%$ \\
$0.0175[0.0146 ; 0.0208]$ & $2.2 \%$ \\
$0.0642[0.0424 ; 0.0927]$ & $2.1 \%$ \\
$0.0326[0.0175 ; 0.0551]$ & $2.1 \%$ \\
$0.0035[0.0010 ; 0.0089]$ & $2.2 \%$ \\
$0.0147[0.0122 ; 0.0177]$ & $2.2 \%$ \\
$0.0000[0.0000 ; 0.0046]$ & $2.1 \%$ \\
$0.0156[0.0051 ; 0.0361]$ & $2.1 \%$ \\
$0.0231[0.0157 ; 0.0329]$ & $2.2 \%$ \\
$0.0092[0.0011 ; 0.0329]$ & $2.0 \%$ \\
$0.0147[0.0068 ; 0.0278]$ & $2.1 \%$ \\
$0.0059[0.0012 ; 0.0171]$ & $2.1 \%$ \\
$0.0019[0.0000 ; 0.0108]$ & $2.1 \%$ \\
$0.0292[0.0118 ; 0.0592]$ & $2.1 \%$ \\
$0.0093[0.0030 ; 0.0217]$ & $2.1 \%$ \\
$0.0233[0.0186 ; 0.0288]$ & $2.2 \%$ \\
$0.0000[0.0000 ; 0.0016]$ & $2.2 \%$ \\
$0.0038[0.0020 ; 0.0066]$ & $2.2 \%$ \\
$0.0000[0.0000 ; 0.0085]$ & $2.1 \%$ \\
$0.0092[0.0002 ; 0.0501]$ & $1.9 \%$ \\
$0.1739[0.1582 ; 0.1905]$ & $2.2 \%$ \\
$0.0409[0.0343 ; 0.0484]$ & $2.2 \%$ \\
$0.1216[0.1186 ; 0.1247]$ & $2.2 \%$ \\
$0.0119[0.0094 ; 0.0149]$ & $2.2 \%$ \\
$0.0300[0.0200 ; 0.0431]$ & $2.2 \%$ \\
$0.0089[0.0057 ; 0.0132]$ & $2.2 \%$ \\
$0.0168[0.0081 ; 0.0307]$ & $2.1 \%$ \\
$0.0064[0.0046 ; 0.0086]$ & $2.2 \%$ \\
$0.0000[0.0000 ; 0.0430]$ & $1.8 \%$ \\
$0.0076[0.0016 ; 0.0221]$ & $2.1 \%$ \\
$0.0122[0.0068 ; 0.0200]$ & $2.2 \%$ \\
$0.0000[0.0000 ; 0.0086]$ & $2.1 \%$ \\
$0.0434[0.0390 ; 0.0482]$ & $2.2 \%$ \\
$0.0350[0.0304 ; 0.0402]$ & $2.2 \%$ \\
$0.0236[0.0149 ; 0.0355]$ & $2.2 \%$ \\
$0.0114[0.0067 ; 0.0183]$ & $2.2 \%$ \\
$0.0021[0.0004 ; 0.0062]$ & $2.2 \%$ \\
$0.0676[0.0412 ; 0.1036]$ & $2.1 \%$ \\
$0.0000[0.0000 ; 0.0081]$ & $2.1 \%$ \\
$0.0000[0.0000 ; 0.0232]$ & $2.0 \%$ \\
$0.0675[0.0566 ; 0.0797]$ & $2.2 \%$ \\
$0.0083[0.0034 ; 0.0171]$ & $2.1 \%$ \\
$0.0392[0.0358 ; 0.0429]$ & $2.2 \%$ \\
& \\
\hline
\end{tabular}

$0.0169[0.0087 ; 0.0277] 100.0 \%$

Fig. 3. Forest plot of prevalence of severe depressive symptoms among Chinese university students amid the COVID-19 pandemic.

association between frequent social media exposure and depressive symptoms in the general population (Gao et al., 2020a).

\section{Cohort-specific prevalence of depressive symptoms}

The higher risk of depressive symptoms in female than in male students during the COVID-19 pandemic is in line with the findings of previous studies with samples of general university students (Li et al., 2018; Gao et al., 2020b; Ismail et al., 2020). This phenomenon could be ascribed to the personality traits of females, such as higher levels of neuroticism/negative emotionality and conscientiousness, in comparison to males (Klein et al., 2011; Weisberg et al., 2011). A meta-analysis of studies comparing the psychopathology between only children and children with siblings in China revealed the small mental health advantage experienced by only child university students in comparison to their peers with siblings, i.e. fewer psychiatric symptoms, including depressive symptoms (Falbo and Hooper, 2015). It seems that this phenomenon also exists in university students affected by the COVID-19 pandemic, i.e. significantly lower rate of depressive symptoms in only child students than in students with siblings, with a small magnitude of difference between the two groups (Cohen's $d=0.17$ ) (Table 2).

One possible explanation for the higher risk of depressive symptoms in overseas than in domestic students is the status of ethnic minority groups in foreign countries (Li et al., 2014). As migrants, overseas students per se have inadequate social support, and this situation worsens owing to the social distancing requirements during the COVID-19 pandemic, potentially increasing the risk of depressive symptoms (Zhong et al., 2015). Due to the higher levels of academic stress in postgraduates than in undergraduates, it is generally believed that postgraduates are at higher risk for depressive symptoms than undergraduates in China (Wang et al., 2019). Similarly, a significantly higher prevalence 
Table 2. Results of meta-analyses of prevalence of depressive symptoms among Chinese university students

\begin{tabular}{|c|c|c|c|c|c|c|c|c|c|}
\hline Subpopulation by variable & $\begin{array}{l}\text { Number } \\
\text { of studies }\end{array}$ & $\begin{array}{l}\text { Sample } \\
\text { size }\end{array}$ & $\begin{array}{l}\text { Number of } \\
\text { depressed } \\
\text { students }\end{array}$ & $\begin{array}{l}\text { Heterogeneity, } \\
\text { I2 (\%) (P) }\end{array}$ & $\begin{array}{l}\text { Pooled } \\
\text { prevalence (95\% } \\
\text { CI), \% }\end{array}$ & $\begin{array}{l}\text { Proportion transformation } \\
\text { approach }\end{array}$ & $z$ & $P$ & $\begin{array}{l}\text { Cohen's } \\
d^{a}\end{array}$ \\
\hline \multicolumn{10}{|l|}{ Overall } \\
\hline Depressive symptoms & 84 & 1292811 & 235330 & $99.9(<0.001)$ & $26.0(23.3,28.9)$ & Freeman-Tukey double arcsine & & & \\
\hline Severe depressive symptoms & 47 & 140423 & 7831 & $99.4(<0.001)$ & $1.69(0.87,2.77)$ & Freeman-Tukey double arcsine & & & \\
\hline \multicolumn{10}{|l|}{ Gender } \\
\hline Male & 27 & 352972 & 70616 & $99.8(<0.001)$ & $28.6(21.4,38.3)$ & Log & & & \\
\hline Female & 28 & 449308 & 106677 & $99.9(<0.001)$ & $30.8(24.3,39.2)$ & Log & 119.34 & $<0.001$ & 0.270 \\
\hline \multicolumn{10}{|l|}{ Ethnic group } \\
\hline Han & 2 & 3079 & 565 & $99.4(<0.001)$ & $21.0(0.64,41.3)$ & Untransformed & & & \\
\hline Minorities & 2 & 537 & 101 & $97.9(<0.001)$ & $21.7(0.00,46.4)$ & Untransformed & 0.66 & 0.321 & 0.032 \\
\hline \multicolumn{10}{|l|}{ Residence place } \\
\hline Urban & 11 & 37382 & 9796 & $99.9(<0.001)$ & $27.4(18.0,41.8)$ & $\log$ & & & \\
\hline Rural & 11 & 47872 & 10487 & $99.9(<0.001)$ & $26.8(16.3,44.2)$ & $\log$ & 6.41 & $<0.001$ & 0.044 \\
\hline \multicolumn{10}{|l|}{ The only-child in the family } \\
\hline Yes & 4 & 1931 & 309 & $98.3(<0.001)$ & $20.7(9.81,43.6)$ & Log & & & \\
\hline No & 4 & 2194 & 419 & $99.2(<0.001)$ & $24.2(10.4,56.5)$ & Log & 5.53 & $<0.001$ & 0.171 \\
\hline \multicolumn{10}{|l|}{ Subject category } \\
\hline Medical & 34 & 33263 & 10717 & $99.5(<0.001)$ & $27.5(21.0,34.6)$ & Arcsine & & & \\
\hline Non-medical & 20 & 32329 & 9181 & $99.7(<0.001)$ & $27.5(20.6,36.7)$ & Log & $<0.001$ & 0.399 & $<0.001$ \\
\hline \multicolumn{10}{|l|}{ Type of students } \\
\hline Oversea students & 2 & 423 & 201 & $97.9(<0.001)$ & $44.5(13.5,75.6)$ & Untransformed & & & \\
\hline Domestic students & 82 & 1292388 & 235129 & $99.9(<0.001)$ & $25.6(22.9,28.3)$ & Freeman-Tukey double arcsine & 12.32 & $<0.001$ & 0.844 \\
\hline \multicolumn{10}{|l|}{ Grade } \\
\hline Undergraduates & 42 & 1183315 & 206398 & $99.9(<0.001)$ & $22.9(19.6,26.3)$ & Freeman-Tukey double arcsine & & & \\
\hline Postgraduates & 14 & 20303 & 4192 & $99.1(<0.001)$ & $29.3(21.6,37.7)$ & Arcsine & 112.14 & $<0.001$ & 1.028 \\
\hline \multicolumn{10}{|c|}{ Geographic location of current residence } \\
\hline Hubei & 9 & 14849 & 3977 & $95.0(<0.001)$ & $27.5(22.8,32.2)$ & Untransformed & & & \\
\hline Non-Hubei & 22 & 768375 & 162354 & $99.5(<0.001)$ & $22.3(17.6,27.9)$ & Logit & 130.35 & $<0.001$ & 1.033 \\
\hline \multicolumn{10}{|l|}{ Location of the university } \\
\hline Hubei & 8 & 23290 & 6455 & $99.3(<0.001)$ & $26.2(19.5,33.6)$ & Freeman-Tukey double arcsine & & & \\
\hline Non-Hubei & 59 & 1190886 & 207920 & $99.9(<0.001)$ & $23.1(19.4,27.2)$ & Logit & 66.74 & $<0.001$ & 0.543 \\
\hline
\end{tabular}




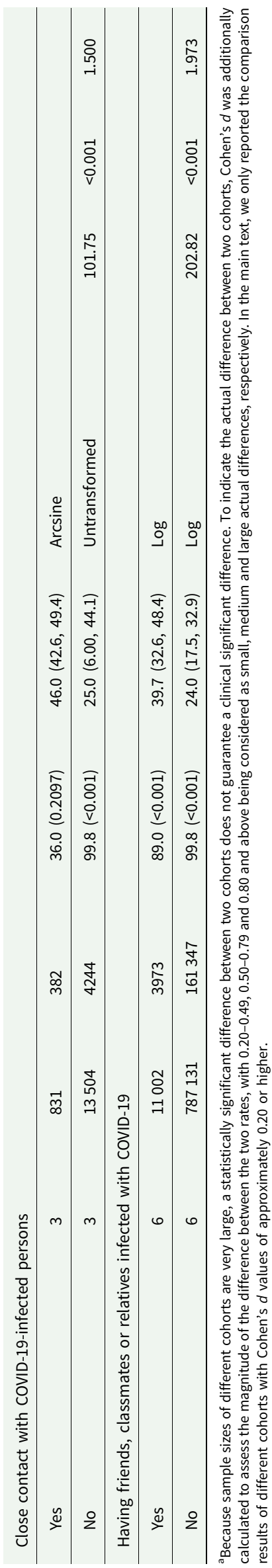

of depressive symptoms in postgraduates than in undergraduates was observed in our study. According to our experiences with some university students from the crisis hotline services during the outbreak period, the negative impact of the COVID-19 pandemic on academic achievement is greater in postgraduates than in undergraduates since undergraduates are able to continue their studies through online courses, but many postgraduates rely on university campus labs to continue their research. Because of the closure of campuses, postgraduates are more likely to be depressed.

Due to Hubei residents' higher risk of infection and provincewide stringent mass quarantine measures, an elevated risk of depressive symptoms in students living in the epicentre relative to that in students living outside the epicentre is expected. Despite having left Hubei before the Spring Festival, students from universities in Hubei had been compulsorily isolated for medical observation in their hometowns and experienced a high level of discrimination and social exclusion due to their potential to spread the COVID-19 virus at the initial stage of the outbreak (He et al., 2020). Therefore, it is reasonable to find significantly higher rates of depressive symptoms in students from universities at the epicentre than in those from universities of provinces other than Hubei in our study.

Studies have reported the significant association of depressive symptoms with having relatives or acquaintances infected with COVID-19 in general populations of both China and Italy during the COVID-19 pandemic (Mazza et al., 2020; Zhong et al., 2020). Consistent with these findings, the rate of depressive symptoms was significantly higher in university students with COVID-19-infected acquaintances or relatives, which may be attributed to these students' high levels of concern about the health of the infected persons. Previous studies have found a greater level of fear of COVID-19 infection in persons who were suspected of having COVID-19, which was in turn associated with a higher risk of depressive symptoms (Koçak et al., 2021; Tsang et al., 2021). For a similar reason, university students with a history of COVID-19 contact exhibited a significantly higher prevalence of depressive symptoms.

\section{Findings from subgroup analysis}

Subgroup analysis revealed a higher prevalence of depressive symptoms in studies with samples with fewer men, which is consistent with the female predominance phenomenon of depression (Albert, 2015). However, what is counterintuitive is the higher risk of depressive symptoms in studies conducted late in the COVID-19 outbreak than that in studies conducted early in the COVID-19 outbreak in the subgroup analysis because the daily number of newly confirmed COVID-19 cases in China peaked during the early stage, and the outbreak was under control during the late stage. Similarly, a two-wave longitudinal study in China found increased severity of depressive symptoms in a cohort of the general population four weeks after the epidemic's peak relative to the initial COVID-19 outbreak (Wang et al., 2020a). We speculate that during the early stage, people may have been shocked by the sudden outbreak, and they focused on safety and physical health. After the outbreak, the negative impacts of the pandemic, including economic loss and unemployment, gradually increased with time, leading people to feel depressed. Because of the problematic methodology of poorly designed studies, i.e. mental health surveys adopting convenience sampling are likely to recruit students having potential needs for mental health 
Fig. 4. Funnel plot of publication bias among the 84 included studies.

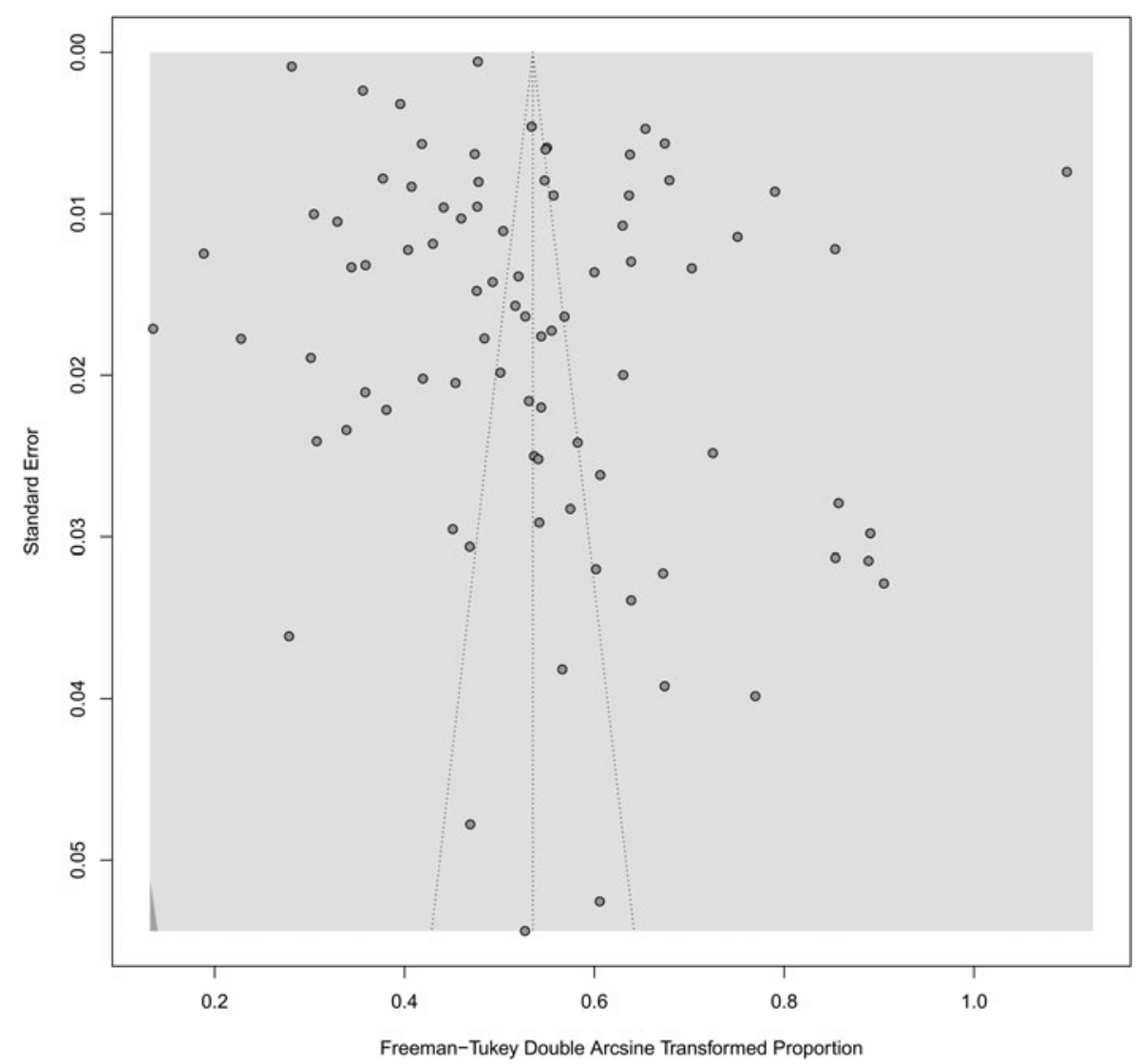

services, a statistically higher prevalence of depressive symptoms in studies with a high level of RoB was found in this study.

\section{Limitations}

This study has some limitations. First, none of the included studies were rated as completely low RoB. Subgroup analysis according to RoB level found a significantly higher prevalence of depressive symptoms in studies with a high level of RoB, so it is possible that the reported overall pooled estimate overestimates the true prevalence. Second, because several included studies used strict criteria to define the presence of depressive symptoms (i.e. PHQ-9 $\geqslant 10$ ), we may have underestimated the prevalence of depressive symptoms. Given the above two limitations, it is difficult to assess the magnitude and direction of bias in the prevalence estimate. Cautions are needed when generalising our findings. Third, even after stratifying the studies, high levels of heterogeneity were still kept within each strata of study in the subgroup analysis, so there remained other factors associated with the risk of depressive symptoms that were not identified. The heterogeneity of the results suggests that further rigorously designed studies using widely accepted assessments of depressive symptoms and representative samples of Chinese university students amid the COVID-19 pandemic are warranted to arrive at accurate estimates. Fourth, because of the small number of studies during the postoutbreak period, longitudinal data are needed to examine the trajectory of depressive symptoms in Chinese university students in the postpandemic era. Fifth, since the sample size of overseas students was relatively small $(n=423)$, the sample representativeness of overseas students may be limited in our study. Finally, patterns of utilisation of mental health services among depressed students are very important for mental health planning and policy-making in the context of the COVID-19 pandemic, but the included studies provided little information on service use.

\section{Implications and conclusions}

In this study, over one out of every four Chinese university students had depressive symptoms, which suggests a high level of mental healthcare need in this population amid the COVID-19 pandemic. Depression takes a high toll on individuals, families and societies, and, in particular, it is a major risk factor for attempted and completed suicide. Given the high prevalence of depressive symptoms, mental health services for this population amid the pandemic should include periodic evaluation of depressive symptoms to ensure early identification of students with severe depressive symptoms or high risk of suicide and psychiatric assessment and treatment when necessary. The higher prevalence rates of depressive symptoms revealed in several cohorts of Chinese university students (i.e. postgraduates, students living in the epicentre and COVID-19 contacts) indicate that cohortspecific prevention programmes, which are probably costeffective, need to be designed.

China is a mental health services resource-poor country, so university managers and staff, including campus psychological counselors, should have a critical role in depression prevention; for example, they could provide expanded social support to students at risk, engage in follow-up care, mental health education and periodic screening of depressed students and promote social 
Table 3. Subgroup analysis of the source of heterogeneity of included studies

\begin{tabular}{|c|c|c|c|c|c|c|c|}
\hline Study characteristics & $\begin{array}{l}\text { Number } \\
\text { of studies }\end{array}$ & $\begin{array}{l}\text { Sample } \\
\text { size }\end{array}$ & $\begin{array}{c}\text { Number of } \\
\text { depressed } \\
\text { students }\end{array}$ & $\begin{array}{l}\text { Heterogeneity, } \\
\qquad 12(\%)(P)\end{array}$ & $\begin{array}{l}\text { Pooled prevalence } \\
(95 \% \mathrm{CI}), \%\end{array}$ & $Q$ & $P$ \\
\hline \multicolumn{8}{|l|}{ Survey period } \\
\hline Early stage of COVID-19 outbreak & 32 & 1214858 & 211065 & $99.9(<0.001)$ & $21.8(18.3,25.5)$ & Reference & \\
\hline Late stage of COVID-19 outbreak & 34 & 50290 & 18286 & $99.6(<0.001)$ & $31.0(24.5,38.0)$ & 5.93 & 0.015 \\
\hline Post-COVID-19 era & 6 & 12881 & 3411 & $99.6(<0.001)$ & $28.9(15.7,44.2)$ & 0.95 & 0.329 \\
\hline Not reported & 12 & 14782 & 2568 & $98.2(<0.001)$ & $22.3(17.2,27.8)$ & 0.03 & 0.869 \\
\hline \multicolumn{8}{|c|}{ Percentage of males among the survey sample } \\
\hline$\geqslant 50 \%$ & 14 & 22866 & 4321 & $98.8(<0.001)$ & $20.6(15.7,26.0)$ & Reference & \\
\hline$<50 \%$ & 65 & 1262658 & 229437 & $99.9(<0.001)$ & $27.3(24.2,30.5)$ & 4.53 & 0.033 \\
\hline Not reported & 5 & 7287 & 1572 & $96.3(<0.001)$ & $24.1(15.5,34.0)$ & 0.45 & 0.500 \\
\hline \multicolumn{8}{|l|}{ Mean/median age (years) } \\
\hline$>20.8$ & 19 & 70547 & 16696 & $99.9(<0.001)$ & $31.4(20.2,43.8)$ & Reference & \\
\hline$\leqslant 20.8$ & 21 & 83904 & 19037 & $99.5(<0.001)$ & $25.7(21.4,30.2)$ & 0.81 & 0.367 \\
\hline Not reported & 44 & 1138360 & 199597 & $99.9(<0.001)$ & $23.9(20.5,27.5)$ & 1.49 & 0.222 \\
\hline \multicolumn{8}{|l|}{ Survey method } \\
\hline Online self-administered & 77 & 1289363 & 234634 & $99.9(<0.001)$ & $26.3(23.5,29.1)$ & & \\
\hline Paper-pencil self-administered & 7 & 3448 & 696 & $95.6(<0.001)$ & $22.3(15.7,29.5)$ & 1.05 & 0.306 \\
\hline \multicolumn{8}{|l|}{ Sampling method } \\
\hline Convenience sampling & 61 & 1170724 & 213585 & $99.9(<0.001)$ & $27.1(23.7,30.7)$ & & \\
\hline Probability sampling & 23 & 122087 & 21745 & $99.4(<0.001)$ & $23.0(19.7,26.4)$ & 2.87 & 0.090 \\
\hline \multicolumn{8}{|l|}{ Assessment } \\
\hline Depression subscale of SCL-90-R & 8 & 9378 & 1215 & $98.4(<0.001)$ & 11. $5(6.6,17.5)$ & Reference & \\
\hline PHQ-9 & 37 & 1189597 & 212581 & $99.9(<0.001)$ & $27.3(23.5,31.2)$ & 17.39 & $<0.001$ \\
\hline CES-D & 7 & 56504 & 10734 & $99.9(<0.001)$ & $40.0(22.4,58.9)$ & 10.07 & 0.002 \\
\hline Depression subscale of PQEEPH & 2 & 3442 & 565 & $57.3(0.130)$ & $16.4(14.5,18.3)$ & 2.42 & 0.120 \\
\hline Depression subscale of DASS- 21 & 7 & 7055 & 1119 & $98.9(<0.001)$ & $21.1(11.7,32.5)$ & 2.81 & 0.094 \\
\hline SDS & 22 & 26201 & 8970 & $99.7(<0.001)$ & $28.4(19.0,38.9)$ & 9.25 & 0.002 \\
\hline Depression subscale of HADS & 1 & 634 & 146 & Not applicable & $23.0(19.8,26.4)$ & 10.45 & 0.001 \\
\hline \multicolumn{8}{|l|}{ Cut-off score of PHQ-9 } \\
\hline$\geqslant 10$ & 5 & 352561 & 29275 & $99.4(<0.001)$ & $15.5(10.3,21.5)$ & & \\
\hline$\geqslant 5$ & 32 & 837036 & 183306 & $99.8(<0.001)$ & $29.2(26.4,32.1)$ & 15.33 & $<0.001$ \\
\hline \multicolumn{8}{|l|}{ RoB score } \\
\hline 7-8 (low) & 11 & 95842 & 15507 & $99.5(<0.001)$ & $20.6(16.4,25.0)$ & Reference & \\
\hline 4-6 (moderate) & 42 & 1156633 & 208692 & $99.9(<0.001)$ & $25.7(21.7,29.9)$ & 2.88 & 0.090 \\
\hline 0-3 (high) & 31 & 40336 & 11131 & $98.8(<0.001)$ & $28.4(24.3,32.7)$ & 6.49 & 0.011 \\
\hline
\end{tabular}

PHQ-9, 9-item Patient Health Questionnaire; DASS-21, Depression, Anxiety and Stress Scale - 21 Items; PQEEPH, Psychological Questionnaires for Emergent Events of Public Health; SCL-90-R, Symptom Checklist-90-Revised; CES-D, Center for Epidemiologic Studies Depression Scale; SDS, Zung's Self-Depression Rating Scale; HADS, Hospital Anxiety and Depression Scale.

connectedness between students. Although the pandemic increases physical distances between staff and students, support services can be easily provided to students via smartphones.

In addition, the $28.9 \%$ prevalence of depressive symptoms during the postoutbreak era in this study (Table 3) and some small new COVID-19 outbreaks in recent months in China suggest the necessity of continuous mental health monitoring and services for Chinese university students during the postoutbreak era. Further rigorous research is also needed to understand the longitudinal changes in depressive symptoms of Chinese university students during the postoutbreak era.

Supplementary material. The supplementary material for this article can be found at https://doi.org/10.1017/S2045796021000202

Data. All the data involved have been included in Tables and Figures of this paper, including supplementary files. 
Financial support. The study was supported by National Key Research and Development Program of China (Grant No.: 2018YFC1314303, PI: XiangRong Zhang) and the National Natural Science Foundation of China (71774060, Bao-Liang Zhong, PI). The funding source had no role in study design; in the collection, analysis and interpretation of data; in the writing of the report; and in the decision to submit the paper for publication.

Conflict of interest. None.

Ethical standards. Not applicable.

\section{References}

Albert PR (2015) Why is depression more prevalent in women? Journal of Psychiatry \& Neuroscience 40, 219-221.

Barendregt JJ, Doi SA, Lee YY, Norman RE and Vos T (2013) Meta-analysis of prevalence. Journal of Epidemiology and Community Health 67, 974-978.

Begg CB and Mazumdar M (1994) Operating characteristics of a rank correlation test for publication bias. Biometrics 50, 1088-1101.

Cao D (2020) Study on the relationship between life stressors and depression of vocational college students during the COVID-19 epidemic. Shaanxi Transport Science \& Education Research 1, 35-38.

Chang J, Yuan Y and Wang D (2020) Mental health status and its influencing factors among college students during the epidemic of COVID-19. Journal of Southern Medical University 40, 171-176.

Chen F and Zhu Z (2021) Mental health status of students in university of science and technology during the peak of the COVID-19 epidemic. Psychology Monthly 16, 20-22.

Chen H, Wen Y, Chen J, Chen Y, Liu B, Lu D, Chen Z, Chen J, Shen Y and Hu J (2020a) Investigation and analysis of mental health status of different types of medical postgraduates during the COVID-19 epidemic. China Higher Medical Education 34, 22-23.

Chen RN, Liang SW, Peng Y, Li XG, Chen JB, Tang SY and Zhao JB (2020b) Mental health status and change in living rhythms among college students in China during the COVID-19 pandemic: a large-scale survey. Journal of Psychosomatic Research 137, 110219.

Chen Y, Chen S, Sun J, Hu J and Shen Y (2020c) Difference in mental health status of different types of residents during COVID-19 epidemic. Chinese Journal of Graduate Medical Education 4, 143-146.

Chen Z, Qi X, Du S, Chen H and Ren Z (2020d) A study on the mental health status of students in the "post-epidemic era": taking some college students in Beijing as examples. Psychology Monthly 15, 5-7.

Chi X, Becker B, Yu Q, Willeit P, Jiao C, Huang L, Hossain M, Grabovac I, Yeung A, Lin J, Veronese N, Wang J, Zhou X, Doig S, Liu X, Carvalho A, Yang L, Xiao T, Zou L, Fusar-Poli P and Solmi M (2020) Prevalence and psychosocial correlates of mental health outcomes among Chinese college students during the coronavirus disease (COVID-19) pandemic. Frontiers in Psychiatry 11, 803.

Cong A, Xiao C, Luan S, Kang L, Yuan J and Liu C (2020) Investigation on the mental health status and risk factors among Chinese overseas students under COVID-19 outbreak (preprint). Research Square. https://doi.org/10. 21203/rs.3.rs-35535/v1.

Dang W, Huang Y, Liu Z and Li S (2004) Analysis of anxiety and depression symptoms and related factors in three universities during SARS epidemic in Beijing. Chinese Journal of Behavioral Medical Science 13, 437-439.

Deng CH, Wang JQ, Zhu LM, Liu HW, Guo Y, Peng XH, Shao JB and Xia W (2020) Association of web-based physical education with mental health of college students in Wuhan during the COVID-19 outbreak: crosssectional survey study. Journal of Medical Internet Research 22, e21301.

Diao J (2007) Adolescent-Parent Conflict and Parental Awareness in Middle School Students' Families (Master). Shanghai, China: East China Normal University.

Dong X (2020) Influence study of COVID-2019 on mental health of normal college students. Psychology Monthly 15, 37-39.

Dong H, Wang T, Wei W, Mei X and Chen J (2020) Investigation and analysis of psychological state of medical postgraduates. Journal of Clinical and Experimental Medicine 19, 1247-1249.
Elmer T and Stadtfeld C (2020) Depressive symptoms are associated with social isolation in face-to-face interaction networks. Scientific Reports 10, 1444.

Falbo T and Hooper SY (2015) China's only children and psychopathology: a quantitative synthesis. American Journal of Orthopsychiatry 85, 259-274.

Feng T (2020) Investigation and analysis on mental health status of college students during the COVID-19 epidemic. Journal of Qingyuan Polytechnic 13, 58-65.

Feng Y, Zong M, Yang Z, Gu W, Dong D and Qiao Z (2020) When altruists cannot help: the influence of altruism on the mental health of university students during the COVID-19 pandemic. Globalization and Health 16, 61.

Gao J, Zheng P, Jia Y, Chen H, Mao Y, Chen S, Wang Y, Fu H and Dai J (2020a) Mental health problems and social media exposure during COVID-19 outbreak. PLoS One 15, e0231924.

Gao Y, Zhang G, Yu H, Sui C and Wu T (2020b) Relationship between children psychological maltreatment and depression among college students: a mediating effect of mental resilience. China Journal of Health Psychology 28, $462-466$.

Han T, Ma W, Gong H, Hu Y, Zhang Y, Zhang C, Yao Z, Fan Y, Zheng Y and Wang $C$ (2020) Investigation and analysis of negative emotion among university students during home quarantine of COVID-19. Journal of Xi'an Jiaotong University (Medical Sciences). http://kns.cnki.net/kcms/detail/61. 1399.R.20200426.0824.002.html.

He J, Chen S, Liu L, Fang Y and Li L (2014) Risk factors for depression and the mechanism among college students in Hangzhou. Chinese Journal of School Health 35, 1008-1010.

He J, He L, Zhou W, Nie X and He M (2020) Discrimination and social exclusion in the outbreak of COVID-19. International Journal of Environmental Research and Public Health 17, 2933.

Hu X (2018) The Relationship between Career Planning Ability, Mental Health and College Adaptation of Undergraduates Under the New College Entrance Examination (Master). Shanghai, China: Shanghai Normal University.

Ismail M, Lee KY, Tanjung AS, Jelani IaA, Latiff RA, Razak HA and Shauki NIA (2020) The prevalence of psychological distress and its association with coping strategies among medical interns in Malaysia: a national-level cross-sectional study. Asia-Pacific Psychiatry. doi: 10.1111/appy.12417.

Ji X, Yu R, Mou M, Chen L, Zhao H, Zhou Q, Deng X and Yang X (2020) Analysis of psychological state in Sichuan area nursing undergraduate's during the epidemic of COVID-19. Medical Education Research and Practice 28, 225-228.

Jiang Y, He L, Meng Y, Liu Z, Zhang T, Zou Y, Zhang D, Jiang X and Zhou S (2020) Mental health status and its influencing factors among unveristy students of preventive medicine speciality during the COVID-19 epidemic. Journal of Xiangnan University (Medical Sciences) 22, 59-62.

Kirkpatrick R and Zang Y (2011) The negative influences of exam-oriented education on Chinese high school students: backwash from classroom to child. Language Testing in Asia 1, 36-45.

Klein DN, Kotov R and Bufferd SJ (2011) Personality and depression: explanatory models and review of the evidence. Annual Review of Clinical Psychology 7, 269-295.

Koçak O, Koçak E and Younis MZ (2021) The psychological consequences of COVID-19 fear and the moderator effects of individuals' underlying illness and witnessing infected friends and family. International Journal of Environmental Research and Public Health 18, 1836.

Lei XY, Xiao LM, Liu YN and Li YM (2016) Prevalence of depression among Chinese university students: a meta-analysis. PLoS One 11, e0153454.

Lei R, Liu Y, Sun L, Yuan Y, Song Q, Lin S, Hu J, Yang X and Ma W (2020) Emotion, sleeping problems and coping styles of only child medical students among novel coronavirus pneumonia: an online survey. World Latest Medicine Information (Electronic Version) 20, 224-226, 232.

Leung DYP, Mak YW, Leung SF, Chiang VCL and Loke AY (2020) Measurement invariances of the PHQ-9 across gender and age groups in Chinese adolescents. Asia-Pacific Psychiatry 12, e12381.

Li K and He Y (2020) Research on the practice mode of psychological education in colleges and universities during the epidemic period: a case study of Jinhua Vocational and Technical College. Vocational Education 19, 50-53. 
Li J, Wang Y, Xiao F and Tech T (2014) East Asian international students and psychological well-being: a systematic review. Journal of International Students 4, 301-313.

Li L, Wan L and Zhao S (2018) Characteristics and influencing factors of depression of college students. Journal of Qiannan Normal University for Nationalities 38, 81-84, 100

Li W, Yang Y, Liu Z-H, Zhao Y-J, Zhang Q, Zhang L, Cheung T and Xiang Y-T (2020a) Progression of mental health services during the COVID-19 outbreak in China. International Journal of Biological Sciences 16, 1732-1738.

Li X, Lv Q, Tang W, Deng W, Zhao L, Meng Y, Guo W and Li T (2020b) Psychological stresses among Chinese university students during the COVID-19 epidemic: the effect of early life adversity on emotional distress. Journal of Affective Disorders 282, 33-38.

Li J, Zhang R, Zou Y, Gu F, Meng J, Gao L and Shen Y (2021) Influencing factors of depressive symptoms in Zhejiang adolescents. Preventive Medicine 33, 139-142.

Lian X, Tan X and Zhang J (2020) Survey of and intervention strategy for mental health problems of university students during the COVID-19 epidemic. Journal of Higher Education 27, 33-36, 40.

Liang J, Cui X and Zhang Y (2020a) Investigation on mental health status of nursing students during COVID-19 epidemic. Journal of Heilongjiang University of Technology 20, 1-4.

Liang Y, Zheng C and Yu H (2020b) A survey and analysis of mental health status of medical postgraduates during the COVID-19 epidemic. China Higher Medical Education 34, 34-35.

Lin X and Xu J (2020) Influence of physical exercise on mental health of college students during the epidemic of COVID-19. Chinese Journal of School Health 41, 1682-1687.

Lin J, Guo T, Becker B, Yu Q, Chen ST, Brendon S, Hossain MM, Cunha PM, Soares FC, Veronese N, Yu JJ, Grabovac I, Smith L, Yeung A, Zou L and $\mathrm{Li} \mathrm{H}(2020 a)$ Depression is associated with moderate-intensity physical activity among college students during the COVID-19 pandemic: differs by activity level, gender and gender role. Psychology Research and Behavior Management 13, 1123-1134.

Lin Z, Lin X and Jiang X (2020b) Research on the relationship between emotion regulation strategies and emotional response of medical students during the COVID-19 epidemic. Straits Science 21, 91-93.

Liu N (2020a) Analysis and guidance of college students' mental health against the epidemic of novel coronavirus pneumonia. Social Sciences Journal of Universities in Shanxi 32, 33-36.

Liu Y (2020b) The establishment of psychological crisis intervention model of college nursing students affected by the epidemic. Vocational Education 13, 32-37.

Liu S, Ma J, Wei P and Jia F (2004) The mental state of medical college student during the period of SARS diffusion. Health Psychology Journal 12, 117-118.

Liu F, Zhou N, Cao H, Fang X, Deng L, Chen W, Lin X, Liu L and Zhao H (2017) Chinese College freshmen's mental health problems and their subsequent help-seeking behaviors: a cohort design (2005-2011). PLoS One 12, $\mathrm{e} 0185531$

Liu J, Zhu Q, Fan W, Makamure J, Zheng C and Wang J (2020a) Online mental health survey in a medical college in China during the COVID-19 outbreak. Frontiers in Psychiatry 11, 459.

Liu S, Yuan Y and Luo B (2020b) Influence of COVID-19 on depression and anxiety of college students and analysis of related factors. Journal of Clinical Research 37, 819-823.

Liu X, Liu J and Zhong X (2020c) Psychological state of college students during COVID-19 epidemic (preprint). SSRN, http://dx.doi.org/10.2139/ssrn. 3552814 .

Luo R (2020) Relationship between social isolation and parent-child conflicts of college students during COVID-19. Journal of Wenzhou Polytechnic 20, 21-25.

Ma H, Yang C, Liu N, Tao J, Zhang L and Gao H (2019) Study on the relationships of depressive symptoms with personality traits and coping styles of undergraduates. Chinese Journal of Health Education 35, 179-181, 191.

Ma L, Wang X and Liao Q (2020a) Effect of stress events of COVID-19 on psychological health of college students. Health Research 40, 257-260.
Ma Z, Zhao J, Li Y, Chen D, Wang T, Zhang Z, Chen Z, Yu Q, Jiang J, Fan F and Liu X (2020b) Mental health problems and correlates among 746217 college students during the coronavirus disease 2019 outbreak in China. Epidemiology and Psychiatric Sciences 29, e181.

Mao S, Luo S, Li F, Zhang B, Wang P, Li M and Wu S (2020) Research on the anxiety and depression status of medical postgraduates in the late epidemic. Journal of Harbin Medical University 54, 560-563.

Marmorstein NR and Iacono WG (2004) Major depression and conduct disorder in youth: associations with parental psychopathology and parentchild conflict. Journal of Child Psychology and Psychiatry 45, 377-386.

Mazza C, Ricci E, Biondi S, Colasanti M, Ferracuti S, Napoli C and Roma P (2020) A nationwide survey of psychological distress among Italian people during the COVID-19 pandemic: immediate psychological responses and associated factors. International Journal of Environmental Research and Public Health 17, 3165.

Munn Z, Moola S, Riitano D and Lisy K (2014) The development of a critical appraisal tool for use in systematic reviews addressing questions of prevalence. International Journal of Health Policy and Management 3, 123-128.

Ni J, Wang F, Liu Y, Wu M, Jiang Y, Zhou Y, Zhou Y and Sha D (2021) Psychological impact of the COVID-19 pandemic on Chinese health care workers: cross-sectional survey study. JMIR Mental Health 8, e23125.

Pan M, Zhang S, Zhou S, Cong T, Tao M, Han Y, Hou Y, Cao P and and Zhen Q (2021) Analysis of related factors and coping styles of college students' mental health under stress. China Journal of Health Psychology 29, 309-313.

Qian Q (2020) Anxiety and depression and their coping strategies in medical students during the COVID-19 epidemic. Leisure 18, 237-238.

Ren L and Edwards CP (2015) Pathways of influence: Chinese parents' expectations, parenting styles, and child social competence. Early Child Development and Care 185, 614-630.

Ren H, Li C and Zhang Q (2020a) Mental health status of university students and the effectiveness of prevention and control strategies in the context of COVID-19 epidemic. Psychology Monthly 15, 135-136.

Ren S, Wang F, Li G, Hou W, Liu J, Hu B and Wei Q (2020b) Investigation on the status of influencing factors for depression and negative emotions of college students in Inner Mongolia during the prevalence of novel coronavirus pneumonia. Journal of Baotou Medical College 36, 70-74.

Ren Y, Chen Y and Cui W (2020c) Analysis of mental health status and influencing factors of nursing interns during COVID-19 outbreak. Journal of Qilu Nursing 26, 8-11.

Rognli EW, Waraan L, Czajkowski NO, Solbakken OA and Aalberg M (2020) Conflict with parents in adolescent depression: associations with parental interpersonal problems and depressive symptoms. Child Psychiatry and Human Development 51, 442-452.

Si M, Su XY, Jiang Y, Wang WJ, Gu X, Ma L, Li J, Zhang SK, Ren ZF, Liu YL and Qiao YL (2020) Psychological impact and associated factors during the containment stage of Covid-19 epidemic among college students in China (preprint). Research Square, https://doi.org/10.21203/rs.3.rs-80603/v1.

Sun Y, Lin SY and Chung KKH (2020) University students' perceived peer support and experienced depressive symptoms during the COVID-19 pandemic: the mediating role of emotional well-being. International Journal of Environmental Research and Public Health 17, 9308.

Sun S, Goldberg S, Lin D, Qiao S and Operario D (2021) Psychiatric symptoms, risk, and protective factors among university students in quarantine during the COVID-19 pandemic in China. Globalization and Health 17, 15.

Tang W, Hu T, Hu B, Jin C, Wang G, Xie C, Chen S and Xu J (2020) Prevalence and correlates of PTSD and depressive symptoms one month after the outbreak of the COVID-19 epidemic in a sample of homequarantined Chinese university students. Journal of Affective Disorders 274, 1-7.

The State Council Information Office of the People's Republic of China (2020) Fighting COVID-19: China in Action [Online]. Beijing: The State Council Information Office of the People's Republic of China. Available at http://www.scio.gov.cn/zfbps/ndhf/42312/Document/1682142/1682142. htm (accessed 17 February 2021).

Tsang S, Avery AR and Duncan GE (2021) Fear and depression linked to COVID-19 exposure a study of adult twins during the COVID-19 pandemic. Psychiatry Research 296, 113699. 
Vaughan CA, Foshee VA and Ennett ST (2010) Protective effects of maternal and peer support on depressive symptoms during adolescence. Journal of Abnormal Child Psychology 38, 261-272.

Wan C and Shao X (2020) Research and investigation of mental health status of vocational college students under COVID-19 epidemic. Heilongjiang Science 11, 144-147.

Wang J and He K (2020) A COVID-19 epidemic reaction and perceived social support of college students. Journal of Yibin University 20, 21-26.

Wang Y and Li Q (2020) Study on the mental health of college students and its influencing factors during the COVID-19 epidemic: based on an investigation of some universities in Sichuan, Yunnan and Chongqing. Journal of Aba Teachers University 37, 109-117.

Wang R, Huang Y, Wang H, Wei X, Wang C, Li Z and Wei R (2019) Study on comorbid anxiety and depression in medical bachelor and postgraduates. Continuing Medical Education 33, 16-18.

Wang C, Pan R, Wan X, Tan Y, Xu L, Mcintyre RS, Choo FN, Tran B, Ho R, Sharma VK and Ho C (2020a) A longitudinal study on the mental health of general population during the COVID-19 epidemic in China. Brain, Behavior, and Immunity 87, 40-48.

Wang K, Wang Q, Liu B, Jia J, Ren H and Chen M (2020b) Mental health of college students of different specialties during the epidemic of coronavirus disease 2019. Journal of Xi'an Jiaotong University (Medical Sciences), http:// kns.cnki.net/kcms/detail/61.1399.R.20200526.1036.002.html.

Wang M, Xie Q and Liu J (2020c) A survey on psychological depression of postgraduates in the epidemic of COVID-19. The Science Education Article Collects 15, 159-161.

Wang W, Wu X and Yu Q (2020d) The effect of survivors' guilt on depression: the mediating role of rumination. Journal of South China Normal University (Social Science Edition) 6, 22-30.

Wang Y, Chen Q, Zhao H and Liu J (2020e) Survey and analysis of mental health status of college students in Wuhan during the post-COVID-19 pandemic. Chinese Journal of Health Psychology, https://kns.cnki.net/kcms/ detail/11.5257.r.20201025.1721.004.html.

Wang ZH, Yang HL, Yang YQ, Liu D, Li ZH, Zhang XR, Zhang YJ, Shen D, Chen PL, Song WQ, Wang XM, Wu XB, Yang XF and Mao C (2020f) Prevalence of anxiety and depression symptom, and the demands for psychological knowledge and interventions in college students during COVID-19 epidemic: a large cross-sectional study. Journal of Affective Disorders 275, 188-193.

Wang J, Ding Y, Jiang Q, Liao C and Li L (2021) Anxiety and depression levels and coping strategies of college students in Anhui Province under the COVID-19. China Journal of Health Psychology 29, 266-271.

Wei J (2020) Mental health problems and coping strategy of university students amid the COVID-19 epidemic. China Journal of Multimedia \& Network Teaching 3, 217-219.

Weisberg YJ, Deyoung CG and Hirsh JB (2011) Gender differences in personality across the ten aspects of the big five. Frontiers in Psychology 2, 178.

Wu R (2019) Suicide Risk of College Students and the Effect of Brief Mediation Training (Master). Shanghai, China: The Second Military Medical University.

Wu B, Tao Z and Han X (2020) Exploration and analysis of college students' psychological health status during the epidemic situation: a case study of Shanghai University of electric power. Psychology Monthly 15, 43-44.

Wu X, Tao S, Zhang Y, Li S, Ma L, Yu Y, Sun G, Li T and Tao F (2021) Geographic distribution of mental health problems among Chinese college students during the COVID-19 pandemic: nationwide, web-based survey study. Journal of Medical Internet Research 23, e23126.

Xiang MQ, Tan XM, Sun J, Yang HY, Zhao XP, Liu L, Hou XH and Hu M (2020) Relationship of physical activity with anxiety and depression symptoms in Chinese college students during the COVID-19 outbreak. Frontiers in Psychology 11, 582436.

Xiao H, Shu W, Li M, Li Z, Tao F, Wu X, Yu Y, Meng H, Vermund SH and Hu Y (2020a) Social distancing among medical students during the 2019 coronavirus disease pandemic in China: disease awareness, anxiety disorder, depression, and behavioral activities. International Journal of Environmental Research and Public Health 17, 5047.

Xiao H, Wang L, Xiao C and Yan H (2020b) Analysis of psychological behavior of college students in Wuhan during the COVID-19 epidemic. Journal of Public Health and Preventive Medicine 31, 7-11.
Xie L, Luo H, Li, M, Ge W, Xing B and Miao Q (2020) The immediate psychological effects of Coronavirus Disease 2019 on medical and non-medical students in China. International Journal of Public Health 65, 1445-1453.

Xin M, Luo S, She R, Yu Y, Li L, Wang S, Ma L, Tao F, Zhang J, Zhao J, Li L, Hu D, Zhang G, Gu J, Lin D, Wang H, Cai Y, Wang Z, You H, Hu G and Lau JT (2020) Negative cognitive and psychological correlates of mandatory quarantine during the initial COVID-19 outbreak in China. American Psychologist 75, 607-617.

Xing B, Ge W, Lu Y, Shu W and Miao Q (2020) A survey of medical students' cognition, psychology and behavior of COVID-19. Health Research 40, 369-372.

Xiong P, Ming WK, Zhang C, Bai J, Luo C, Cao W, Zhang F and Tao Q (2020) Factors influencing mental health among Chinese medical and nonmedical students in the early stage of COVID-19 pandemic (preprint) Research Square, https://doi.org/10.21203/rs.3.rs-139929/v1.

Xu W and Li G (2020) Analysis of mental health status and differences of independent college students during the COVID-19. Psychology Monthly 15, 83-84.

Yan S, Zheng J, Lin Z, Xie Q, Wang S and Zheng J (2020) The role of coping disposition on the relationship between personality and negative emotion in medical students during the COVID-19 epidemic. Journal of Mu Dan Jiang Medical University 41, 170-174.

Yang X, Gao L, Zhang S, Zhang L, Zhang L, Qi M and Chen J (2020a) Professional identity of Chinese medical students during the COVID-19 pandemic (preprint). Research Square, https:/doi.org/10.21203/rs.3.rs-144141/v1.

Yang Y, Wang Y, Li S, Lei X and Yang Y (2020b) Psychological responses and related factors of college students in Shaanxi during COVID-19 outbreak. Chinese Journal of School Health 41, 664-667.

Yao X, Xu Y, Zhang M, Bu J, Cao Y and Wang S (2020) A mental health survey of 84 military college students during the COVID-19 epidemic. Practical Journal of Medicine \& Pharmacology 37, 580-582, 590.

Yi H, Peng Z, Zhang, L and Kong S (2020a) Mental health of art students in Lingnan Normal University during the period of COVID-19 outbreak. China Journal of Health Psychology 28, 1491-1495.

Yi Z, Sun Y and Xie J (2020b) Investigation report on mental health status of medical students during the outbreak of New coronavirus pneumonia. World Latest Medicine Information (Electronic Version) 20, 263-264, 266.

Yu G, Fang S and Luo P (2020) Research on the mental health status of college students during the COVID-19 epidemic and its educational implications. The Modern Education Journal 38, 53-63, 71.

Yu M, Tian F, Cui Q and Wu H (2021) Prevalence and its associated factors of depressive symptoms among Chinese college students during the COVID-19 pandemic. BMC Psychiatry 21, 66.

Zhan J, Sun S, Xie L, Wen Y and Fu J (2020) Medical students' mental health, professional pride, and intention to work in the front line during coronavirus disease 2019 pandemic. Journal of Central South University (Medical Sciences) 45, 648-655.

Zhang CL, Xu YM and Zhong BL (2020a) The association between smoking and loneliness among Chinese university freshmen. Annals of Translational Medicine 8, 649 .

Zhang J, Zeng J, Luo J, Zou X and Gu J (2020b) Epidemiological survey of the COVID-19 epidemic-related knowledge, behaviors and psychology status among college students and their family members. Modern Preventive Medicine 47, 3754-3758.

Zhang L, Liu X, Guo S, Zhang Y, Liang W, Li K and Ni Z (2020c) 2019 novel coronavirus (2019-nCoV) associated mental health among medical students and non-medical students. China Journal of Health Psychology, https://kns. cnki.net/kcms/detail/11.5257.R.20200612.0905.002.html.

Zhang S, Gao L, Yang X, Zhang L, Qi M and Chen J (2020d) Relationship between the COVID-19 exposure and depression and anxiety: mediating role of social support. Chinese Journal of School Health 41, 657-660.

Zhang T, Meng Y, Deng D, Huang H, Tang L, You S, Jiang Y and Duan Y (2020e) A survey on the mental health of medical students facing the public health emergency. Journal of Xiangnan University (Medical Sciences) 22, 51-55.

Zhang X, Jia W and Duan L (2020f) An investigation and analysis of psychological status of 1486 medical students in the the period of COVID-19. Journal of Inner Mongolia Medical University 42, 128-130.

Zhang X, Sui X and Chang R (2020g) The relationship between social support and anxiety and depression during the COVID-19 epidemic: a case study of 
college students. Journal of Liaoning Normal University (Social Science Edition) 43, 83-87.

Zhang XD, Jing HQ, Wang Y, Li KH, Zhao D, Yu HY and Zhang LY (2020h) The psychological responses and associated factors of Chinese college students during COVID-19 outbreak in February (preprint). Research Square, https://doi.org/10.21203/rs.3.rs-103598/v1.

Zhao Y and Hu W (2020) A survey on sleep and mental health of homequarantined college students during the COVID-19 epidemic. Shangqing $12,224,264$.

Zhao X, Selman R and Haste H (2015) Academic stress in Chinese schools and a proposed preventive intervention program. Cogent Education 2, 1000477.

Zhao J, Chen Z, Liang S and Sheng Q (2019) The mediating effect of the regulatory emotional self-efficacy from postgraduates in medicine on their depression and suicide. Chinese Journal of Special Education 26, 72-77.

Zhao B, Kong F, Aung MN, Yuasa M and Nam EW (2020a) Novel coronavirus (COVID-19) knowledge, precaution practice, and associated depression symptoms among university students in Korea, China, and Japan. International Journal of Environmental Research and Public Health 17, 6671.
Zhao B, Kong F and Nam EW (2020b) Knowledge, preventative practices and depression of Chinese university students in Korea and China during the COVID-19 pandemic: an online cross-sectional study (preprint). Research Square, https://doi.org/10.21203/rs.3.rs-48092/v1.

Zhao J, Zhang S, Zheng G, Lian C and Huang L (2020c) A survey on emotion and sleep qualty of university students during the COVID-19 epidemic. Blooming Season 21, 96-97.

Zhong BL, Liu TB, Chan SS, Jin D, Hu CY, Dai J and Chiu HF (2015) Prevalence and correlates of major depressive disorder among rural-to-urban migrant workers in Shenzhen, China. Journal of Affective Disorders 183, 1-9.

Zhong BL, Zhou DY, He MF, Li Y, Li WT, Chee HN, Xiang YT and Chiu HFK (2020) Mental health problems, needs, and service use among people living within and outside Wuhan during the COVID-19 epidemic in China. Annals of Translational Medicine 8, 1392.

Zhou SJ, Qi M, Wang LL, Yang XJ, Zhang LG, Yang R and Chen J (2020) Mental health problems and related factors in Chinese university students during the COVID-19 pandemic (preprint). Research Square, https://doi. org/10.21203/rs.3.rs-58137/v1. 